IEEE

COMMUNICATIONS

ISURVEYS

The Electronic Magazine of

Original Peer-Reviewed Survey Articles

www.comsoc.org/pubs/surveys

\title{
A SURVEY OF QOS ROUTING SOLUTIONS FOR MOBILE AD HOC NETWORKS
}

\author{
LAJOS HANZO II AND RAHIM TAFAZOLLI, UNIVERSITY OF SURREY
}

\begin{abstract}
In mobile ad hoc networks (MANETs), the provision of quality of service (QoS) guarantees is much more challenging than in wireline networks, mainly due to node mobility, multihop communications, contention for channel access, and a lack of central coordination. QoS guarantees are required by most multimedia and other time- or error-sensitive applications. The difficulties in the provision of such guarantees have limited the usefulness of MANETs. However, in the last decade, much research attention has focused on providing QoS assurances in MANET protocols. The QoS routing protocol is an integral part of any QoS solution since its function is to ascertain which nodes, if any, are able to serve applications' requirements. Consequently, it also plays a crucial role in data session admission control. This document offers an up-to-date survey of most major contributions to the pool of QoS routing solutions for MANETs published in the period 1997-2006. We include a thorough overview of QoS routing metrics, resources, and factors affecting performance and classify the protocols found in the literature. We also summarize their operation and describe their interactions with the medium access control (MAC) protocol, where applicable. This provides the reader with insight into their differences and allows us to highlight trends in protocol design and identify areas for future research.
\end{abstract}

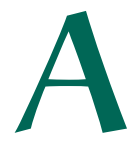

$\mathrm{t}$ the time of writing this article, the field of mobile ad hoc networks (MANETs) [1] has been recognized as an area of research in its own right for more than ten years. Much hope for spontaneous and robust wireless communications has been placed in MANETs due to their decentralized, self-configuring, and dynamic nature, which avoids the need for an expensive base station infrastructure. In the mid-to-late 1990s, research focused mainly on designing distributed and dynamic communications protocols for sharing the wireless channel and for discovering routes between mobile devices. The aim of these protocols was to provide a basic best-effort level of service to ensure network operation in the face of an unpredictable and shared wireless communication medium and to maintain a network topology view and routes in the face of failing links and mobile devices.

Despite the vast array of technological solutions for MANETs, their practical implementation and use in the real world has been limited so far. Since entertainment and other multimedia services are usually what drive the mass uptake of a technology, it follows that to truly realize the potential of MANETs, they must be able to deliver such services, for which best-effort protocols are not adequate.

This is because multimedia applications often have stringent time- and reliability-sensitive service requirements, which the network must cater to. As a consequence, especially in the past five or six years, focus has shifted from best-effort services to the provision of higher and better-defined QoS in MANET research. QoS routing protocols play a major part in a QoS mechanism, since it is their task to find which nodes, if any, can serve an application's requirements. Therefore, the QoS routing protocol also plays a major part in session admission control (SAC), since that is dependent on the discovery of a route that can support the requested QoS. Alternatively, some QoS routing solutions may not attempt to serve applications' requirements directly, rather they may seek to improve all-round QoS under particular metrics.

The majority of the solutions proposed in the literature till now have focused on providing QoS based on two metrics: throughput and delay. Of these, the more common is throughput. This is probably because assured throughput is somewhat of a "lowest common denominator" requirement; most voice or video applications require some level of guaranteed 
throughput in addition to their other constraints. However, many other metrics are also used to quantify QoS and in this work we cover most of them and provide examples of their use. The remainder of this article is structured as follows. In the following section we discuss related work in terms of QoS routing surveys and summarize their main points. This is followed by a brief review of the challenges posed by the provision of QoS on the MANET environment. Next, we present an overview of commonly employed QoS routing metrics, the factors affecting QoS protocol performance, the network resources consumable by applications, and some of the tradeoffs involved in protocol design. We then continue by describing some methods of classifying QoS routing solutions, in order to organize the many candidate solutions.

Following this, we summarize the operation, key features and major advantages and drawbacks of a selection of QoS routing protocols proposed in the literature. We focus on journal articles and peer-reviewed conferences, thereby hopefully extracting the most useful and important subset of the candidate solutions. According to one of the classification methods in the fifth section, we discuss the proposals under various headings. Firstly, the sixth section provides some examples of QoS routing protocols that rely on contentionfree MAC. The seventh section does the same for solutions operating with a contended MAC. Finally, methods that do not rely on any specific kind of MAC are presented. Under each section, we group protocols into different types of approaches, although for some approaches, only one example is provided. We then discuss our findings and the observed trends in the field of QoS routing. Then, future work areas are identified according to our own findings in this survey, before a summary is given in the final section.

Note that throughout this article, we consistently employ the term "(data) session" as opposed to some other authors who prefer to use "call" or "(data) flow."

\section{RELATED WORK}

A literature search already yields several overviews and surveys of QoS routing issues and solutions. However, the last one was published in early 2004, and in this article we cover many proposals published since then. Also, we select some different and less well known protocols for inclusion in our survey as a means of highlighting alternative approaches to QoS routing. We additionally provide a more thorough background, especially in terms of metrics and design trade-offs, and devise new means of classification. Consequently, the trends and future work identified also differ greatly in this document. A fairly comprehensive overview of the state of the field of QoS in networking in 1999 was provided by Chen in [2]. Chakrabarti and Mishra [3] later summarized the important QoS-related issues in MANETs that were in focus around 2001 , and the issues that required further attention. This article was updated and expanded in 2004 [4]. Their conclusions highlighted several significant points:

- Many of the underlying algorithmic problems, such as multiconstraint routing, have been shown to be NP-complete.

- QoS and, indeed, best-effort routing can only be successfully achieved if the network is combinatorially stable. This means that the nodes are not moving faster than routing updates can propagate.

- Different techniques are required for QoS provisioning when the network size becomes very large, since QoS state updates would take a relatively long time to propagate to distant nodes.

- There is a trade-off between QoS provisioning and mini- mization of power utilization.

Several areas of future work were also identified:

- Admission control policies and protocols require further attention

- QoS robustness

- QoS routing protocol security against, for example, denial-of-service attacks - the combination of security and QoS provisioning

- Study of QoS preservation under failure conditions

- QoS support for multicast applications

In 2004, Al-Karaki and Kamal published a detailed overview [5] about the state of, and the development trends in, the field of QoS routing. They highlighted the following areas as requiring further research attention, where some may be duplicated from [4]:

- Accommodating multiple classes of traffic, in particular, ensuring that lower-class traffic is not starved of network resources in the presence of realtime traffic; additionally, the inclusion of preemptive scheduling

- Preservation of QoS guarantees under various failure conditions

- The use of position-determination systems such as GPS for aiding QoS routing

- Prioritization of control packets above data packets in QoS routing

- Use of more "realistic" mobility models, as opposed to the overly simplistic ones often employed in simulation studies (for example, random way-point)

- Quantifying the impact of cross-layer integration

- Interaction of MANETs with the Internet and the impact on QoS routing thereof

- Security in the QoS routing protocol to prevent malicious retransmission, snooping, and redirection of packets, for example

- The impact of and solutions to network partitioning in the context of QoS routing

- The effect of introducing devices that are heterogeneous in terms of their capacity and capabilities

Many of those considerations, such as security and multicast routing, are beyond the scope of this article. In this work we focus on the essence of QoS routing, which is the discovery of routes that can service data sessions and session admission control, which depends on the routes discovered.

In [5] many of the QoS routing solutions existing in early 2004 were discussed and categorized into the following types of approaches: flat (all nodes play an equal role), hierarchical (some nodes are local cluster heads for example), positionbased (utilize location information), and power-aware (take battery usage and residual charge into consideration) QoS routing.

Finally, a thorough overview of the more widely accepted MAC and routing solutions for providing better QoS was presented in [6]. Reddy et al. also provided various classifications of QoS solutions, as discussed below.

\section{Problems Facing the Provision of QOS IN MANETS}

The following is a summary of the major challenges to providing QoS guarantees in MANETs.

Unreliable Wireless Channel - the wireless channel is prone to bit errors due to interference from other transmissions, thermal noise, shadowing, and multipath fading effects [7]. This makes it impossible to provide hard packet delivery ratio or link longevity guarantees. 
Node Mobility - the nodes in a MANET may move completely independently and randomly as far as the communications protocols are concerned. This means that topology information has a limited lifetime and must be updated frequently to allow data packets to be routed to their destinations. Again, this invalidates any hard packet delivery ratio or link stability guarantees. Furthermore, a QoS state which is link- or node position dependent must be updated with a frequency that increases with node mobility.

An important general assumption must also be stated here: for any routing protocol to be able to function properly, the rate of topology change must not be greater than the rate of state information propagation. Otherwise, the routing information will always be stale and routing will be inefficient or could even fail completely. This applies equally to QoS state and QoS route information. A network that satisfies this condition is said to be combinatorially stable [3].

Lack of Centralized Control - the major advantage of an ad hoc network is that it may be set up spontaneously, without planning, and its members can change dynamically. This makes it difficult to provide any form of centralized control. As such, communications protocols which utilize only locally available state and operate in a completely distributed manner are preferred [8]. This generally increases an algorithm's overhead and complexity, as QoS state information must be disseminated efficiently.

Channel Contention - In order to discover network topology, nodes in a MANET must communicate on a common channel. However, this introduces the problems of interference and channel contention. For peer-to-peer data communications these can be avoided in various ways. One way is to attempt global clock synchronization and use a TDMA-based system where each node may transmit at a predefined time. This is difficult to achieve due to the lack of a central controller, node mobility and the complexity and overhead involved [9]. Other ways are to use a different frequency band or spreading code (as in CDMA) for each transmitter. This requires a distributed channel selection mechanism as well as the dissemination of channel information. However data communications take place, without a central controller, some setup, new neighbor discovery and control operations must take place on a common contended channel. Indeed, avoiding the aforementioned complications, much MANET research, as well as the currently most popular wireless ad hoc networking technology $(802.11 \mathrm{x})$ is based on fully-contended access to a common channel, that is, with Carrier-Sense Multiple Access with Collision Avoidance (CSMA/CA).

However, CSMA/CA greatly complicates the calculation of potential throughput and packet delay, compared to TDMAbased approaches. This is because nodes must also take into account the traffic at all nodes within their carrier sensing range. Furthermore, the possibility of collisions also arises. Collisions waste channel capacity, as well as node battery energy, increase delay, and can degrade the packet delivery ratio.

Finally, the well-understood hidden node [10] and exposed node [11] problems are a further consequence of channel contention. These problems are even more pronounced when we consider that nodes may interfere with transmissions outside of their transmission range $[9,12,13]$, since receivers are able to detect a signal at a much greater distance than that at which they can decode its information.

Limited Device Resources - to some extent this is an historical limitation, since mobile devices are becoming increasingly powerful and capable. However, it still holds true that such devices generally have less computational power, less memory, and a limited (battery) power supply, compared to devices such as desktop computers typically employed in wired networks. This factor has a major impact on the provision of QoS assurances, since low memory capacity limits the amount of QoS state that can be stored, necessitating more frequent updates, which incur greater overhead. Additionally, QoS routing generally incurs a greater overhead than best-effort routing in the first place, due to the extra information being disseminated. These factors lead to a higher drain on mobile nodes' limited battery power supply. Finally, within the pool of QoS routing problems, many are NP-complete [3], and thus complicated heuristics are required for solving them, which may place an undue strain on mobile nodes' less-powerful processors.

\section{QOS RouTING PROTOCOL DESIGN CONSIDERATIONS}

\section{Metrics USED to SPECIFY QOS ReQUIREMENTS}

The following is a sample of the metrics commonly used by applications to specify QoS requirements to the routing protocol. Consequently, they may be used as constraints on route discovery and selection. Each metric is followed by a reference which provides an example of a protocol that employs the metric as a QoS constraint.

- Minimum Required Throughput or Capacity (b/s) - the desired application data throughput. For an example of QoS routing using this metric/constraint, see [14].

- Maximum Tolerable Delay (s) - usually defined as the maximum tolerable end-to-end (source to destination) delay for data packets [15].

- Maximum Tolerable Delay Jitter - one widely accepted definition of this metric is the difference between the upper bound on end-to-end delay and the absolute minimum delay [16]. The former incorporates the queuing delay at each node and the latter is determined by the propagation delay and the transmission time of a packet. The transmission time between two nodes is simply the packet size in bits/the channel capacity. This metric can also be expressed as delay variance [17].

- Maximum Tolerable Packet Loss Ratio (PLR) - the acceptable percentage of total packets sent, which are not received by the transport or higher layer agent at the packet's final destination node [18].

An application may typically request a particular quality of service by specifying its requirements in terms of one or more of the above metrics. For example, it may require a guaranteed throughput of $500 \mathrm{~kb} / \mathrm{s}$ and a maximum packet delay of $50 \mathrm{~ms}$. In most cases, the QoS protocol should only admit this data session into the network if it can provide the requested service. The mechanism by which this decision is made is termed session admission control (SAC) or just admission control.

\section{Node States AND Metrics EMPloyed for ROUTE SELECTION}

This section lists many of the metrics commonly employed by routing protocols for path evaluation and selection in order to improve all-round QoS or to meet the specific requirements of application data sessions. Many of these metrics, especially those measured at lower layers, are not directly interesting to the application layer; hence, they are listed in this section. 
However, they all, at least indirectly, affect the QoS experienced by a data session.

\section{Network Layer Metrics}

- Achievable throughput or residual capacity (b/s) - the achievable data throughput of a path or node. The achievable throughput or residual capacity is often termed "available bandwidth" in the literature; we prefer to reserve the use of the word "bandwidth" for quantifying the size of frequency bands in $\mathrm{Hz}$. For an example of QoS routing using this metric/constraint, see [14].

- End-to-end delay(s) - the measured end-to-end delay on a path [15].

- Node buffer space - the number of packets in a node's transmission buffer plays a major part in determining the amount of delay a packet traveling through that node will suffer (for example, see [19]).

- Delay jitter(s) or variance - the measured delay jitter on a path. See the previous section for a definition.

- Packet loss ratio (PLR) (percent) - the percentage of total packets sent, which is not received by the transport or higher layer agent at the packet's final destination node.

- Energy expended per packet (J) [20].

- Route lifetime(s) - the statistically calculated expected lifetime of a route, which can depend on node mobility as well as node battery charges (see [21]).

\section{Link and MAC Layer Metrics}

- MAC delay - the time taken to transmit a packet between two nodes in a contention-based MAC, including the total time deferred and the time to acknowledge the data [22]. This provides a good indication of the amount of traffic at the relevant nodes.

- Link reliability or frame delivery ratio - the statistically calculated chance (percentage) of a packet or frame being transmitted over a link and correctly decoded at the receiver. See $[23,24]$ for examples of routing protocols employing this metric for path selection.

- Link stability(s) - the predicted lifetime of a link [21].

- Node relative mobility/stability - can be measured as the ratio of the number of neighbors that change over a fixed period to the number that remain the same [25]. For example, if all of the node's neighbors are the same over a fixed period, that node is completely stable in that period, relative to its neighbors. We list this as a link-layer metric, since neighbor discovery is usually performed at that layer.

\section{Physical Layer Metrics}

- Signal-to-interference ratio (SIR) - although a physical layer metric, the received SIR at a destination node can be used as a routing metric that shows link quality, via cross-layer communication. An example is given in [26].

- Bit error rate (BER) - closely related to SIR, this value determines the level of error correction and/or number of retransmissions required over a "link" and has a major impact on the link's reliability metric and on energy consumption. From another perspective, the BER is a consequence of the SIR between two nodes. For an example, see [27].

- Node residual battery charge or cost [20]. Examples are given in [23, 28].

QoS metrics such as the above can be classified as either additive, concave, or multiplicative metrics, based on their mathematical properties [6]. Additive metrics are defined as

$$
\sum_{i=1}^{n} L_{i}(m)
$$

over path $P$ of length $n$, where $L_{i}(m)$ is the value of the metric $m$ over link $L_{i}$ and $L_{i} \in P$. The value of a concave metric $C_{m}$ is defined as the minimum value of that metric over a path, that is, $C_{m}=\min \left(L_{i}(m)\right)$. Finally, a multiplicative metric $M_{m}$ is calculated by taking the product of the values along a path, that is,

$$
M_{m}=\prod_{i=1}^{n} L_{i}(m)
$$

Thus, end-to-end delay for example, is an additive metric, since it is cumulative over the whole path. Available channel capacity is a concave metric, since we are only interested in the bottleneck: the minimum value on the path. Finally, path reliability is a multiplicative metric, since the reliabilities of each link in the path must be multiplied together to compute the chance of delivering the packet via a given route (assuming that the MAC layer retransmissions have been considered in the reliability value, or that there are no retransmissions for example, for broadcast packets).

\section{Protocol Evaluation Metrics}

The following metrics may be used to evaluate a QoS routing protocol's performance.

\section{Transport/Application Layer}

- Session acceptance/blocking ratio - the percentage of application data sessions (or transport layer connections) that are admitted into or rejected from the network. The value of this metric reflects both the effectiveness of the QoS protocols as well as conditions outside of their control, such as channel quality.

- Session completion/dropping ratio - this metric represents the percentage of applications that were successfully/ unsuccessfully served after being admitted to the network. For example, if a VoIP session is accepted and the session is completed properly (by the users hanging up) and not aborted (dropped) due to route failure or any other error, then that counts as a completed session.

\section{Network Layer}

- Network throughput (b/s) - the amount of data traffic the entire network carried to its destination in one second.

- Per-node throughput (b/s) - the average throughput achieved by a single node.

- Route discovery delay(s) for reactive protocols - a measure of the effectiveness of reactive protocols, that is, on average, what is the delay between a route request being issued and a reply with a valid route being received. In some cases, this may also be referred to as the session establishment time (SET).

- Normalized routing load (NRL) — the ratio of routing packets transmitted to data packets received at the destination. This gives a measure of the operating cost and efficiency of the routing protocol. An example is given in [29].

- Network lifetime(s) - may be defined as the time until network partitioning occurs due to node failure [20], or the time until a specified proportion of nodes fail. This measure indicates a protocol's energy-efficiency and load balancing ability.

- Average node lifetime(s) - see [20]. 
- Routing energy efficiency $(\%)=\left(E_{\text {data }}\right) /\left(E_{\text {total }}\right) \times 100$, where $E_{\text {data }}$ and $E_{\text {total }}$ are the energy consumed for the transmission and reception of useful data bits, and the total energy consumed in communicating data packets plus routing headers and control packets, respectively.

\section{MAC Layer}

- Normalized MAC load - similar to the NRL, this represents the ratio of bits sent as MAC control frames to the bits of user data frames transmitted. An example is given in [29].

- MAC energy efficiency - ratio of energy used for sending data bits to the total energy expended for data plus MAC headers and control frames.

\section{Factors Affecting QoS Protocol Performance}

When evaluating the performance of QoS protocols, a number of factors have a major impact on the results. Some of these parameters are a particular manifestation of characteristics of the MANET environment. They define the "scenario," whether in a simulation or in real life, and can be summarized as follows:

- Node mobility - this factor generally encompasses several parameters: the nodes' maximum and minimum speeds, speed pattern, and pause time. The node's speed pattern determines whether the node moves at uniform speed at all times or whether it is constantly varying, and also how it accelerates, for example, uniformly or exponentially with time. The pause time determines the length of time nodes remain stationary between each period of movement. Together with maximum and minimum speed, this parameter determines how often the network topology changes and thus how often network state information must be updated. This parameter has been the focus of many studies, for example, [29, 30].

- Network size - since QoS state has to be gathered or disseminated in some way for routing decisions to be made, the larger the network, the more difficult this becomes in terms of update latency and message overhead. This is the same as with all network state information, such as that used in best-effort protocols [8].

- Number, type and data rate of traffic sources - intuitively, a smaller number of traffic sources results in fewer routes being required and vice versa. Traffic sources can be constant bit rate (CBR) or may generate bits or packets at a rate that varies with time according to the Poisson distribution, or any other mathematical model. The maximum data rate affects the number of packets in the network and hence the network load. All of these factors affect performance significantly [29];

- Node transmission power - some nodes may have the ability to vary their transmission power. This is important, since at a higher power, nodes have more direct neighbors and hence connectivity increases, but the interference between nodes does as well. Transmission power control can also result in unidirectional links between nodes, which can affect the performance of routing protocols. This factor has also been studied extensively, for example, in [31-33].

- Channel characteristics - as detailed above, there are many reasons for the wireless channel being unreliable, that is, many reasons why bits, and hence data packets, may not be delivered correctly. These all affect the network's ability to provide QoS.

\section{Network Resources Required in Order to Provide QOS}

Another question that arises in this section is: what do we mean by "network resources?" Taken literally, a resource is anything that is required in order to perform a task and which is consumed during performance. Therefore, the following is a list of network resources:

- Node computing time - while mobile devices are being manufactured with increasingly powerful processors, they are still limited in computing power, especially when they must not only run the applications, but also the protocols required to support the network and the applications. However, this is probably the least critical resource, as communication protocols usually do not place a heavy burden on the processor.

- Node battery charge - some might argue that this is the most critical resource, since if a node's battery is drained, it cannot function at all. Node failures can also cause network partitioning, leading to a complete network failure and no service provisioning at all. Hence, poweraware and energy efficient MAC and routing protocols have received a great deal of research attention (see [20, 33 and references therein). However, these efforts are beyond the immediate scope of this article.

- Node buffer space (memory) - almost inevitably, at some point during a network's operation, more than one node will be transmitting at once, or there may be no known route to another device. In either of these cases data packets must be buffered while awaiting transmission. Furthermore, when the buffers are full, any newly arriving packets must be dropped, contributing to the packet loss rate;

- Channel capacity - taken literally, this is measured in bps and affects data throughput, and indirectly, delay, and hence a host of other metrics too. However, since all nodes must share the transmission medium, we must somehow express the fraction of the medium's total capacity that is granted for each node's use. The way to express this depends on the MAC layer technique employed. In a purely contention-based MAC, "transmission opportunities" may be envisioned, although no node can be guaranteed channel access, merely granted it with a certain probability. In a Time Division Multiple Access (TDMA)-based solution, channel capacity is expressed in timeslots. Similarly, in FDMA, it is frequency bands, and in spread spectrum techniques, spreading codes. Since, in MANETs, nodes must communicate on the same channel to discover network topology, FDMA and spread spectrum techniques are only employed if there is a separate signaling channel over which to allocate channels to pairs of communicating nodes. The majority of QoS routing solutions in the literature rely on single-channel MAC protocols and are thus contention- or TDMAbased, as we show in this work.

\section{DESIGN TRADE-OFFS}

This section discusses some of the common trade-offs involved in QoS routing protocol design.

Proactive vs. Reactive vs. Hybrid Route Discovery and State Dissemination - We actually refer to two problems under one heading. Firstly, should routes be discovered proactively or on-demand? Secondly, how should the QoS state required for path selection be discovered? If both the route 
and QoS state discovery mechanisms are proactive, then the session establishment time is greatly reduced from an application's point of view. Also, a proactive protocol is largely unaffected by an increase in the fraction of nodes acting as data sources, since routes to all destinations are maintained anyway. However, a large overhead is incurred in keeping routes and state up-to-date, especially in highly mobile scenarios. Additionally, such a mechanism does not scale well with an increasing number of nodes. These are well known problems of proactive protocols [8].

A major advantage of discovering QoS state proactively surfaces in situations where different applications specify their requirements with different metrics. As long as it is decided which QoS states to keep up-to-date, a route may be computed from the routing table based on any QoS metric, without the need for a separate discovery process for each metric for example, see [34].

A purely reactive routing solution avoids the potential wastage of channel capacity and energy by not discovering routes and QoS states which are not currently needed. However, a discovery delay is incurred when an application requires a route to a destination.

A hybrid route discovery approach usually involves defining zones around each node within which proactive route discovery takes place (for example, see [25]). Interzone routing is performed on-demand, eliminating the scaling problems of purely proactive protocols, while intrazone routing enjoys the benefits of routes being readily available.

Hybrid route discovery/state discovery schemes are also feasible. One possibility is where the routes themselves are discovered proactively, but the QoS state is only sought when a QoS-constrained data session is to be admitted (for example, see $[15,35])$. Another possibility is a completely hybrid approach where the QoS state discovery follows the proactive/reactive intrazone/interzone nature of route discovery.

Capacity vs. Delay - It has been shown [36, 37] that in MANETs, capacity can be traded off with packet delay. If delay constraints are relaxed, then the capacity of the network can be increased by exploiting multi-user diversity [36]. More specifically, if delay is not constrained, a source can split the packets of a session and send them to many different neighbors. These neighbors then forward the packets onto the destination when they move into its transmission range. This scheme has been shown to improve throughput, since far fewer intermediate nodes are transmitting packets and causing interference, but incurs the cost of greatly increased delay [36].

Another strategy is to improve delay by increasing redundancy, at the cost of network capacity utilization efficiency [37]. If multiple copies of a packet are forwarded on multiple paths, it has been shown that the destination receives the packet with less delay on average. On the other hand, more network capacity is consumed in sending duplicate packets [37]. Clearly, increased redundancy also reduces the protocol's energy efficiency.

Packet Loss Ratio vs. Capacity and Energy Efficiency In a similar way to the trade-off between delay and capacity, PLR can also be traded off against capacity. Increasing the redundancy by sending multiple copies of packets over different routes results in a higher chance of the destination receiving a copy, but reduces the useful capacity of the network. This technique can be more useful in sensor networks where data are often broadcast without a reliable handshaking protocol being employed at the MAC layer. Once again, redundancy also increases the energy expended per packet.
Energy Consumption vs. Responsiveness and Accuracy of QoS State Information - Routing can only be accurate if the frequency of neighbor discovery is high enough to reflect frequent topological changes. However, a high responsiveness to change comes at an increased energy cost [38]. If we consider QoS routing, this trade-off between accuracy and energy consumption is even more acute, since not only the topology view, but the QoS state information also requires frequent updating so as to enable accurate QoS routing decisions to be made.

Transmission Power Control: Long vs. Short Hops Varying the transmission power to adjust the number of hops required to forward a packet to its destination, can yield many advantages and drawbacks. This has often been called the "long hops versus short hops dilemma." For a detailed discussion of this topic, see [39]. Another question is whether protocol designers should assume the use of transmission power control (TPC) at all. Assuming TPC constrains the type of devices that can be employed, since not all nodes may be equipped with radios with TPC capability. Furthermore, employing TPC can often result in unidirectional links. For example, a node $\mathrm{X}$ may be able to transmit to a node $\mathrm{Y}$, but $Y$ cannot reply, since it is using a lower transmission power, unless it knows the distance to $\mathrm{X}$ and can calculate the transmission power required to reach it.

Global Goals vs. Individual Requirements - From a network designer's point of view, the goal is usually to please as many users as possible by providing an all-round high QoS. Another goal is to increase the network lifetime by spreading the battery usage to avoid node failures and network partitioning. However, each individual user or data session has its own specific requirements and, to satisfy the user, the network must match their requirements.

In more complicated scenarios, an application may specify a variety of QoS constraints. For example, it may specify maximum tolerable values for PLR as well as packet delay. In this case, we desire the routing protocol to find a stable path with a light traffic load. However, from a network lifetime point of view, a path that has the least cost (under some residual battery charge-dependent metric) is preferred. Our goal of low delay matches the aim of load balancing, although the path with the least traffic may not be a stable path and it may also have nodes with the least battery charge remaining. In this case, we clearly have a conflict between our various requirements. A protocol designer must decide how to address this trade-off.

\section{Protocol Classification}

In [5], QoS routing protocols are classified chiefly by their:

- Treatment of network topology (flat, hierarchical, or location-aware)

- Approach to route discovery (proactive, reactive, hybrid, or predictive)

On the other hand, in [6] they are classified in three different ways, based upon:

- The interaction between the route discovery and QoS provisioning mechanism (coupled or decoupled)

- The interaction with the MAC layer (either independent or dependent)

- Again, on the approach to route discovery

In this article we elaborate on the MAC protocol interaction classification, by considering three classes of QoS routing solutions: 


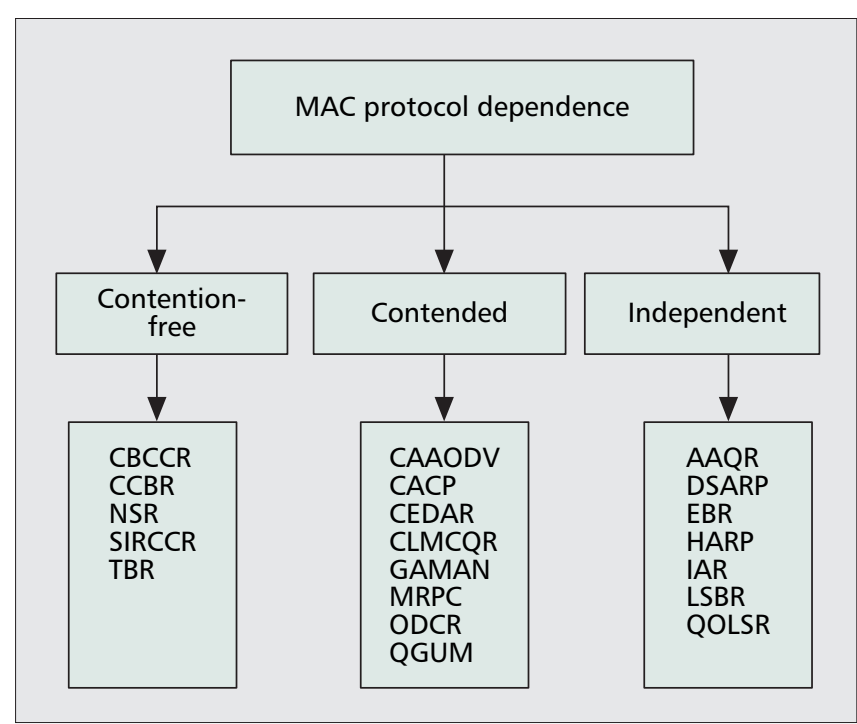

Figure 1. Classification of QoS routing protocols based on MAC layer dependence. There are three categories: 1) the protocol's operation depends on an underlying contention-free MAC protocol, 2) it can operate with a contended MAC protocol, 3) it is completely independent of the MAC protocol.

1 Those that rely on accurately-quantified resource (commonly channel capacity) availability and resource reservation, and therefore require a contention-free MAC solution such as TDMA. Such protocols are able to provide what we term pseudo-hard QoS. Hard QoS guarantees can only be provided in a wired network, where there are no unpredictable channel conditions and node movements. In the solutions that employ a contention-free MAC, the QoS guarantees provided are essentially hard, except for when channel fluctuations or node failures or movements occur, and hence the term "pseudo-hard." Due to these unpredictable conditions, a MANET is not a suitable environment for providing truly hard QoS guarantees.

2 Those that rely only on a contended MAC protocol and therefore only on the available resources or achievable performance to be statistically estimated. Such protocols typically use these estimations to provide statistical or soft guarantees. Implicit resource reservation may still be performed, by not admitting data sessions which are likely to degrade the QoS of previously admitted ones. However, all guarantees are based on contended and unpredictable channel access or are given only with a certain probability and are thus inherently soft.

3 Those that do not require any MAC layer interaction at all and are thus independent from the MAC protocol. Such protocols cannot offer any type of QoS guarantees that rely on a certain level of channel access. They typically estimate node or link states and attempt to route using those nodes and links for which more favorable conditions exist. However, the achievable level of performance is usually not quantified or is only relative and therefore no promises can be made to applications. The aim of such protocols is typically to foster a better average QoS for all packets according to one or more metrics. This comes often at the cost of trade-offs with other aspects of performance (above), increased complexity, extra message overhead, or limited applicability.

In this article we classify and summarize the operation of 20 different QoS routing solution proposals published in highquality literature in the period 1997-2006. This allows us to highlight the variety of approaches investigated, as well as to observe the trends in the field.

Figure 1 illustrates the classification of the 20 protocols based on MAC protocol dependence. The following key applies to the figures in this section: AAQR - Application Aware QoS Routing [17], CAAODV - Contention-Aware Ad hoc On-Demand Distance Vector routing [13], CACP Contention-aware Admission Control Protocol [9], CBCCR - Clustering-based Channel Capacity Routing [40], CCBR Channel Capacity-Based Routing [14], CEDAR - Core Extraction Distributed Ad hoc Routing [41], CLMCQR Cross Layer Multi-Constraint QoS Routing [22], DSARP Delay-Sensitive Adaptive Routing Protocol [19], EBR Entropy-Based Routing [42], GAMAN — Genetic Algorithmbased routing for Mobile Ad hoc Networks [24], HARP Hybrid Ad hoc Routing Protocol [25], IAR — InterferenceAware Routing [43], LSBR — Link Stability-Based Routing [21], MRPC - Maximum Residual Packet Capacity routing [23], NSR — Node State Routing [34], ODCR — On-Demand Delay-Constrained Routing [35], QGUM - QoS-GPSR (Greedy Perimeter Stateless Routing) for Ultra-Wideband (UWB) MANETs [18], QOLSR — QoS Optimized Link State Routing [44], SIRCCR - SIR and Channel Capacity-Based Routing [26], TBR - Ticket-Based Routing [15]. Table 1 and Table 2 summarize the salient features of the 20 protocols whose operation we discuss in later sections.

Classifying based on the QoS metric(s) employed for path evaluation and selection is also possible. However, this classification is not as simple, since many protocols utilize several metrics. In Fig. 2, we have chosen to provide a list of the more popular routing metrics down the centre of the diagram and the protocols are shown either side for increased spatial clarity. A line connects each metric to every protocol which uses it for routing. This illustrates which metrics are more popular by the number of protocols they are linked to. It also shows which protocols utilize a single metric and which ones implement multiconstraint QoS routing. A special case is TBR which is designed to consider two metrics, but not simultaneously; therefore, it is not a multiconstraint routing protocol, and is represented twice: once for each metric. NSR is also a special case. The designers explain how it can be provide an assured throughput service, but it also acts as a framework for routing based on any other metric.

\section{Protocols Relying on CONTENTION-FrEE MAC}

\section{QOS ROUTING IN A CDMA OVER TDMA NETWORK}

The problem that first concerned QoS routing protocol designers was that of discovering paths that satisfy a session's throughput requirement. This was due to the fact that assured throughput seemed to be the lowest common denominator among multimedia data sessions' requirements. Since throughput depends largely on a node gaining sufficient transmission opportunities at the MAC layer, the first part of the solution is to define measures of transmission opportunities, that is, the channel capacity available to a node. Following this, a mechanism is required for estimating the achievable throughput on a path, utilizing the knowledge of the available channel capacity at each forwarding node. Finally, this information can be used to perform session admission control, by only admitting data sessions for which a path with adequate throughput capability has been found.

An early channel-capacity estimation scheme for mobile wireless networks (so-called at the time), was presented in [40]. The authors proposed that a clustering scheme is used to 


\begin{tabular}{|c|c|c|c|c|c|}
\hline Protocol & $\begin{array}{l}\text { QoS assurances } \\
\text { provided }\end{array}$ & $\begin{array}{l}\text { Network/Node } \\
\text { information utilized }\end{array}$ & $\begin{array}{l}\text { Type of QoS } \\
\text { guarantees }\end{array}$ & $\begin{array}{l}\text { MAC protocol function- } \\
\text { ality assumptions }\end{array}$ & $\begin{array}{l}\text { Other } \\
\text { assumptions }\end{array}$ \\
\hline AAQR & $\begin{array}{l}\text { Bounded delay and } \\
\text { jitter; assured } \\
\text { throughput }\end{array}$ & $\begin{array}{l}\text { Packet transmission } \\
\text { delays; session } \\
\text { throughput requirements }\end{array}$ & Soft & None & $\begin{array}{l}\text { Real-time transport } \\
\text { protocol }\end{array}$ \\
\hline CAAODV & Assured throughput & Channel idle time ratio & Soft & $\begin{array}{l}802.11 \text { DCF; channel idle } \\
\text { time estimation }\end{array}$ & AODV routing \\
\hline CACP & Assured throughput & Channel idle time ratio & Soft & $\begin{array}{l}802.11 \text { DCF; channel idle } \\
\text { time estimation }\end{array}$ & Source-routing \\
\hline CBCCR & Assured throughput & Time slot schedule & Pseudo-hard & $\begin{array}{l}\text { CDMA over TDMA; } \\
\text { resource reservation }\end{array}$ & DSDV routing \\
\hline CLMCQR & $\begin{array}{l}\text { Assured throughput, } \\
\text { bounded delay and } \\
\text { packet dropping rate }\end{array}$ & $\begin{array}{l}\text { MAC delay; channel idle } \\
\text { time ratio; link reliability }\end{array}$ & Soft & $\begin{array}{l}\text { Statistical estimation of } \\
\text { the utilized information }\end{array}$ & \\
\hline DSARP & $\begin{array}{l}\text { Reduced delay jitter; } \\
\text { bounded delay }\end{array}$ & Buffer fullness & Soft & None & \\
\hline EBR & $\begin{array}{l}\text { Improved link and } \\
\text { path longevity }\end{array}$ & $\begin{array}{l}\text { Node relative positions } \\
\text { and velocities }\end{array}$ & $\begin{array}{l}\text { No guarantees, } \\
\text { per packet QoS } \\
\text { improvement }\end{array}$ & None & $\begin{array}{l}\text { Relative location } \\
\text { awareness; relative } \\
\text { speed awareness; } \\
\text { source-routing }\end{array}$ \\
\hline
\end{tabular}

Table 1. QoS routing protocol salient features part 1/2.

group nodes and that each cluster employs a different spreading code under a CDMA scheme. Within clusters, the channel was time-slotted to deterministically allocate channel access opportunities for each node. This allows channel capacity to be measured in terms of timeslots. Furthermore, timeslots may be reserved as a way of promising channel capacity to individual data sessions.

The achievable throughput on a link (link capacity) is then determined by the set of common free slots between a transmitter-receiver pair. Note that a general assumption in MANET design is that a node cannot transmit and receive at the same time, since these actions utilize the same frequency band. Therefore, separate timeslots must be employed for these operations. Figure 3 illustrates an example, which is explained later in this section.

With this constraint, the calculation of available channel capacity and the scheduling of free slots between transmitterreceiver pairs on a route are known to be NP-complete problems [40]. In the proposed scheme, this difficulty is alleviated by the use of clustering; gateway nodes between clusters utilize a different spreading code for each cluster and thus avoid the chance of having common free slots with upstream and downstream neighbors. Furthermore, the slot scheduling within a cluster is solved by the cluster head, avoiding the need for a distributed solution. The achievable throughput on a path is then determined by the minimum of the link capacities on the path.

This achievable throughput information is used to augment the classical DSDV routing protocol [45] to perform QoS routing. Timeslots are reserved at nodes by the first arriving data packet and reservations are released when no data packets are received for a certain number of frames.

The ideas in [40] were taken further by Lin and Liu in [14], wherein they devised a detailed algorithm for calculating a path's residual traffic capacity, seemingly filling in the gaps in detail left by [40]. Similar to the aforementioned work, they propose using a CDMA over TDMA network. The channel is time-slotted accordingly, but several communicating pairs can share a timeslot by employing different spreading codes. A path's capacity is expressed in terms of free timeslots. Route discovery is based again on DSDV [45]. Routing updates are used to refresh the "free slot" information in routing tables. The proposed algorithm first calculates the best combination of free slots on the path for maximum throughput and then attempts to reserve them for a particular data session.

In brief, the algorithm deals with nodes in groups of three. Consider the example in Fig. 3, where nodes A, B, and C are intermediate nodes on a path. Below each node we show the timeslots that were free prior to a data session being admitted. In this case, the same six slots were free at each node. At a first trivial glance it appears that the path capacity is six slots. However, if node A transmits to B in slots 1 and 2, as shown with the dark shading, node B must use those two slots for receiving (shaded light gray) and thus cannot use those for transmitting. Say then that B forwards the received traffic to $\mathrm{C}$ in slots 3 and 4 . Node $\mathrm{C}$ must also not transmit in slots 1 and 2 for fear of interfering with B's reception from A at 


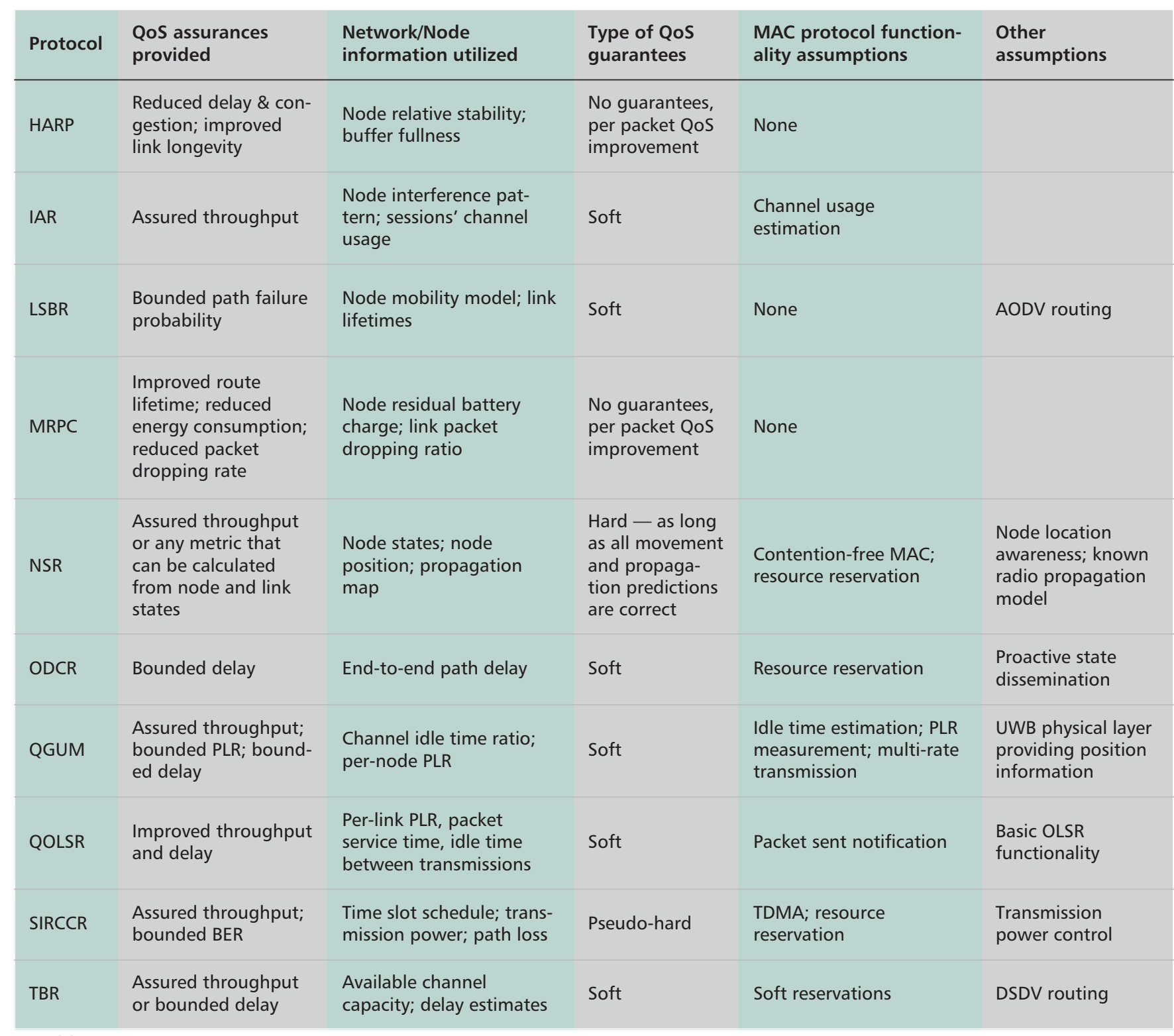

Table 2. QoS routing protocol salient features part 2/2.

those times. Therefore, C may only transmit in slots 5 and 6 . This example illustrates that nodes must have some common free slots to communicate, but if all nodes have the same set of free slots, the efficiency of utilization is not very high. In Fig. 3's example, the effective path capacity usable by a new session is only two slots, despite six being initially free at each node. Once the available timeslots and path capacity have been determined, reservation signaling takes place to reserve the necessary timeslots for satisfying the requesting session's throughput requirement.

The two described schemes offer a clear-cut definition of path capacity in terms of timeslots and allow a routing protocol to provide throughput guarantees to application data sessions by reserving these slots. However, this comes at the cost of many assumptions. First of all, assuming a CDMA network assumes that each group of nodes is assigned a different spreading code. These must either be statically assigned at network start-up, or dynamically assigned. The former mechanism does not deal with nodes/clusters leaving/joining the network, which is one of the most basic characteristics of ad hoc networks. The latter scheme assumes that there is some entity for assigning spreading codes, which is against the ad hoc design principle of not relying on centralized control. Either way, these studies $[14,40]$ do not discuss how code allocation would be achieved.

A second assumption is that of time-slotting. For each frame to begin at the same time at each node, the network must be globally synchronized. Synchronization signaling incurs extra overhead, and as stated in previous work [6,9], in the face of mobility this becomes practically unfeasible. Furthermore, timeslot assignments must be continually updated as nodes move, and sessions are admitted or completed.

Since these designs were published, new TDMA-based MAC protocol designs have come to fruition, such as the IEEE 802.15.3 standard [46]. However, this protocol is designed for use in wireless personal area networks where every node is in range of a controller which provides the timeslot schedule. Thus, it is not suitable for wider-area MANETs. The story is the same with related protocols such as 802.15.4.

The conclusion is that there is currently no ideal feasible solution for implementing TDMA in a multihop MANET environment. We detail other protocols that rely on such a network in order to highlight their other properties which are useful from a design point of view. 


\section{TICKET-BASED MULTIPATH ROUTING}

Chen and Nahrstedt proposed a QoS routing protocol aimed at reducing the QoS route discovery overhead while providing throughput and delay guarantees, in [15]. The main novelty of their approach was in the method of searching for QoS paths. First of all, a proactive protocol, such as DSDV [45] is assumed to keep routing tables up-to-date, with minimum delay, bottleneck throughput and minimum hop to each destination. When a QoS-constrained path is required for a data session, probes are issued by the source node, which are used to discover and reserve resources on a path.

Each probe is assigned a number of tickets and each ticket represents the permission to search one path. The more stringent the delay or throughput requirements of the session, the greater the number of tickets issued. Each intermediate node uses its routing table to decide which neighbors to forward the probe to and with how many of the remaining tickets. Neighbors through which a lower delay or higher achievable throughput (depending on type of search being performed) to the destination is estimated, are assigned more tickets. So, for example, in Fig. 4 the source sends a probe with three tickets, which splits at the second node. Two tickets are issued to the bottom path since it is deemed to have a higher chance of satisfying the delay requirement. Due to the nature of MANETs, the state information is not assumed to be precise and therefore, each delay and bottleneck channel capacity estimate is assumed to be within a range of the estimate, rather than considering the value accurate.

Eventually all probes reach the destination, allowing it to select the most suitable path. It then makes soft reservations by sending a probe back to the source. This probe also sets the incoming and outgoing links for the connection in each node's connections table, setting up a soft connection state. The reservations and states expire when data is not forwarded via that virtual connection for a certain period of time, hence the terms soft reservation/state.

Speaking in its favor, this protocol can handle sessions with either a delay or throughput constraint. When such a constrained path is required, flooding is avoided via the ticket mechanism, while at the same time ensuring that more paths are searched when requirements are stringent, increasing the chance of finding a suitable route. Imprecise state information is also tolerated. However, the method has several drawbacks. Firstly, the protocol used to maintain routing tables for guiding the search probes is proactive, requiring periodic updates, thus incurring a large overhead and not scaling well with network size. Secondly, the study [15] mentions that a TDMA/CDMA MAC is assumed to take care of channel capacity reservation, which has the drawbacks discussed in the previous section.

\section{ON-DEMAND SIR AND BANDWIDTH-GUARANTEED ROUTING With TRansmit Power Assignment}

A much more recent proposal for a TDMA-based QoS routing protocol is presented in [26]. Again, channel capacity is

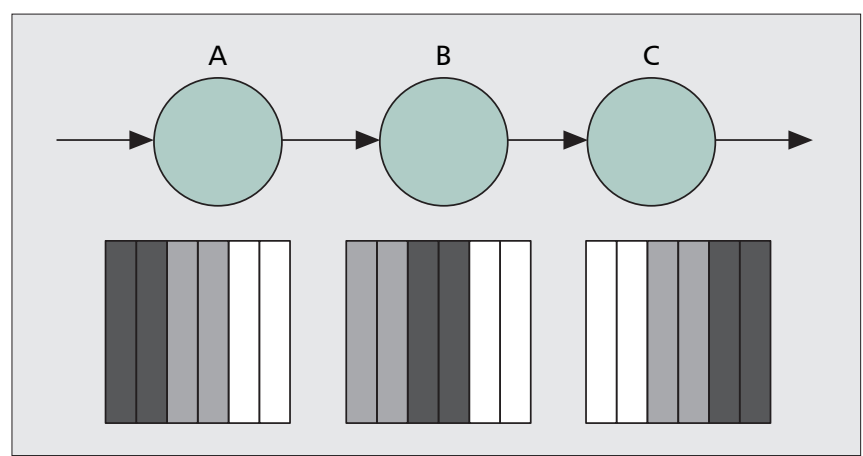

Figure 3. Time slot scheduling example. Dark shading indicates a slot is used for transmitting, and light shading for receiving.

expressed in terms of timeslots. However, an interesting characteristic of this protocol is that it aims to concurrently satisfy not only an application's throughput requirement, but also its bit error rate (BER) constraint. It aims to achieve the latter by assigning adequate transmit power to produce the necessary signal to interference ratio (SIR) between a transmitter and receiver pair, thereby providing a sufficiently low BER. This is in contrast to the general trend in previous candidate solutions, which aimed merely to satisfy a single QoS constraint at any one time. 


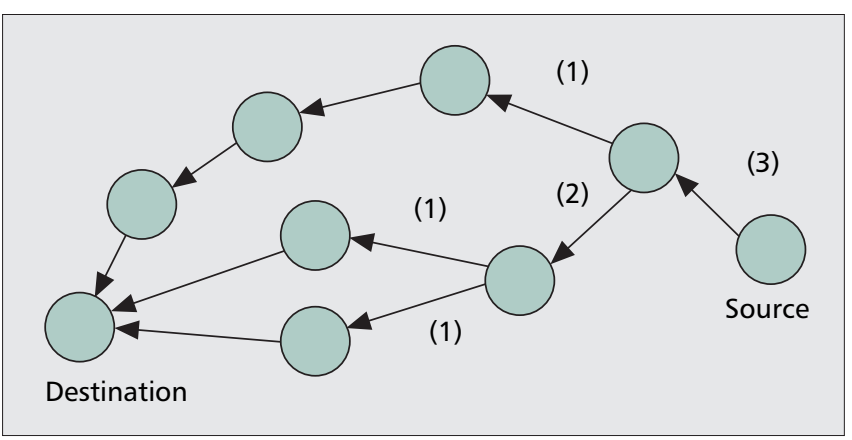

Figure 4. A simple network topology showing a possible ticketbased routing operating scenario. The source issues a probe with three tickets, which then splits as shown. The number of tickets assigned to a path is denoted by the number in brackets. Although the QoS states are not shown, the protocol operates by assigning more tickets to those paths which have a higher likelihood of satisfying the QoS constraints (delay or throughput).

The protocol is on-demand and in essence, follows a similar reactive route discovery strategy to classic reactive routing protocols, such as DSR [47]. An advantage of this protocol is that it gathers multiple routes between a source and destination and allows them to cooperatively satisfy a data stream's throughput requirement. However, only paths that fulfill the SIR requirement on every link qualify as valid routes; the maximum achievable SIR is limited by the maximum transmit power.

Time is split into frames with a control and data phase, each containing several timeslots. In the control phase, each node has a specified slot and uses this to broadcast data phase slot synchronization, slot assignment, and power management information. This broadcast is made at a predefined power level, for example, full power. The received power can be measured and knowing the transmit power, the path loss can be calculated. From this, it is possible to calculate the received SIR. This in turn leads to an estimation for the required link gain and thus the required power at the transmitter, $p_{j-1}^{(i) e s t}$, where $j$ is the current node in the path and $i$ is the timeslot index.

When a route is required, a RReq is broadcast by the source and is received by direct neighbors. The RReq contains the number of timeslots and SIR requirements. Timeslots at the current node must be idle and not used for receiving, to be considered for reservation. Slots for which $p_{j-1}^{(i) e s t}$ is lower, are preferred. As long as one free slot exists, the node is appended to a list in the RReq packet, along with the required power estimate for the transmitter for that particular transmission slot. The destination eventually receives multiple RReqs, hence the need for only one free slot on each path, since multiple paths can cooperatively serve the throughput requirement. It returns RReps to the source along the discovered paths, which deliver the estimated power information so that the correct power can be set in the relevant transmission timeslots. Figure 5 provides an example of an established virtual connection where two paths serve a data session.

This proposal is a good example of a common simplistic approach to multiconstraint QoS routing: one constraint is used merely as a filter, to remove paths which are below a threshold value under that metric. There is no attempt to optimize over multiple metrics. However, this problem has been shown to be NP-complete in many cases [2] (for example, when the metrics are additive [48]), and thus heuristic solution methods are a topic for future research. Additionally, as before, the assumption of a global clock synchronization, which is a prerequisite of a time-slotted system limits the usefulness of this proposal.

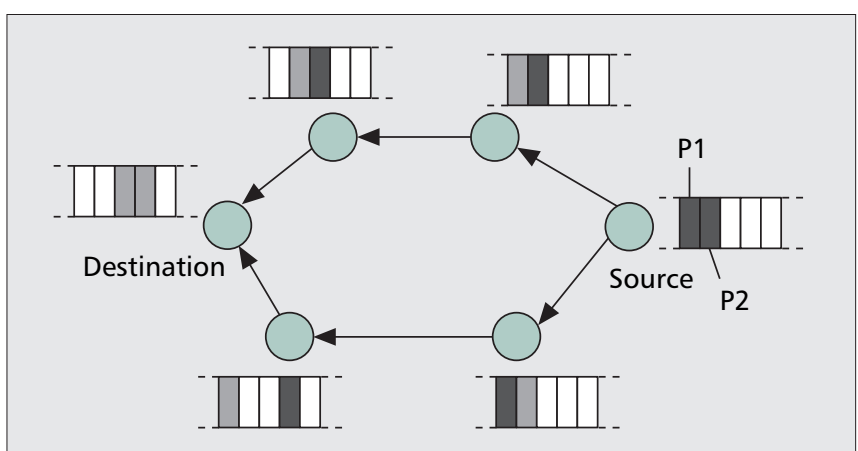

Figure 5. A simple example of the operation of SIR and throughput-guaranteed routing. A section of each node's time slot schedule is shown next to it. Dark shading indicates a slot used for transmission, and light shading, for reception. Unshaded slots are used by other data sessions. In this example, the throughput requirement of the source for its data session is two time slots. The route discovery and time slot assignment phase is over and at the source, slots 1 and 2 have been assigned for transmission. However, each of the two possible next hops have only two slots spare, and one must be used for receiving the source's transmission. The two available paths are used to serve the session's throughput requirement cooperatively, by dedicating one time slot each to transmission. The labels P1 and P2 illustrate the fact that different transmission powers are used in each time slot. As in previous TDMA examples, forwarding nodes must be careful not to transmit in a slot in which their upstream node is receiving.

\section{Node State Routing}

An interesting proposal is discussed in [34]. The authors suggest that the approach taken by most QoS routing protocol designers, of adapting the wireline networking paradigm to ad hoc networks, is wrong. According to this paradigm, nodes are connected by physical entities called links and routing should be performed based on disseminating the state of these links. However, the authors stress that a correct wireless paradigm is one that realizes that communicating node pairs are not connected by a shielded link, rather they share a geographical space and the frequency spectrum with all other communicating pairs in their vicinity. This is clearly true and it follows that links cannot be considered independently of each other. To circumvent this problem, [34] describes node state routing (NSR).

In NSR, each node maintains all potentially useful state information about itself and the space around it, in its routing table. This includes readily-available states such as its IP address, packet queue size and battery charge. However, to avoid relying on link state propagation, NSR requires position awareness via a system such as GPS. This provides more states such as the node's current location, relative speed and direction of movement. Furthermore, it is assumed that nodes can estimate the path loss to neighboring nodes, using a preprogrammed propagation model and knowledge of the node positions. This allows connectivity to be inferred rather than "links" being discovered. Using the aforementioned states, it is also possible to predict connectivity between nodes, whereas in most other protocols, links must be discovered.

In order to perform routing functions, nodes must periodically advertise their states to neighbors. Neighbors should further advertise selected states of their neighbors, for example, only those that have changed beyond a threshold. Using the states of its neighbors, a node may then calculate metrics that may be conceived as link metrics, except that measurements at both "ends" of the link can be taken into account. Moreover, since node states are readily available, they can be used to calculate QoS routes as required. 


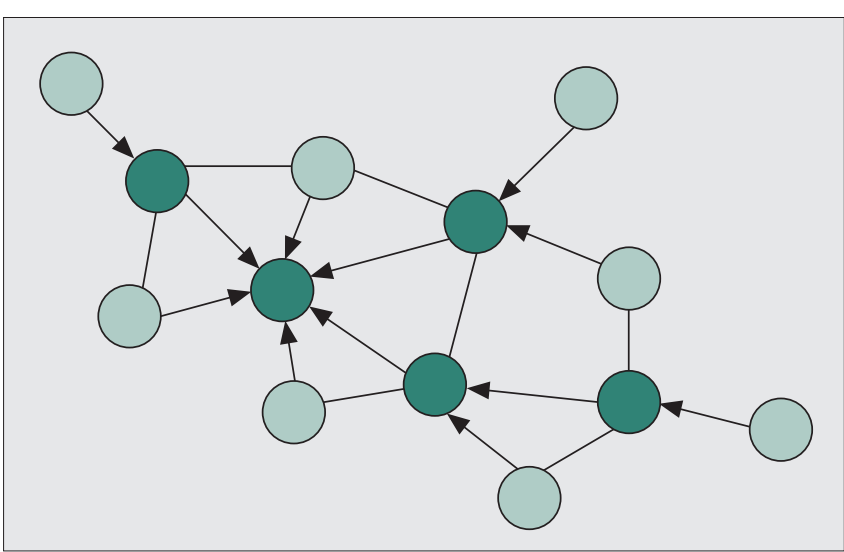

Figure 6. A simple example topology showing a possible core network found by CEDAR. The shaded circles represent core nodes, while the unshaded ones stand for non-core nodes. The core is set up by each node selecting a dominator from among its neighbours. The dominator is initially the neighbour node with the highest degree of connectivity, whose identity is learned through beaconing. A node joins the core if it is selected by at least one node as dominator. The core evolves as each node finally selects the neighbour with the most dominatees to be its dominator. In this gure, the arrows point from each node to its dominator.

As opposed to most other QoS routing protocols, the node states allow different QoS metrics to be considered for each requesting session, without rediscovering routes. A route can be calculated from the propagation map at each node, and its lifetime can be estimated. This approach shows huge potential for practical multiconstraint QoS routing in the future. Furthermore, since link states are not used, there is no need to update several link states when a single node moves, as in other protocols. Instead, only that one node's state needs to be updated in neighbors' state tables.

Despite its many advantages, NSR also has several drawbacks. First and foremost, it relies on accurate location awareness, which limits its usefulness to devices that are capable of being equipped with GPS receivers or such. Secondly, as described in [34], throughput-constrained routing depends on a TDMA-based MAC protocol for capacity reservation and throughput guarantees to be made. Thirdly, the node state updating mechanism is necessarily proactive, which can incur a high overhead and result in poor scaling with network size. However, the authors insist that flooding of states is avoided by propagating only a subset of states to further neighbors and only those that have changed by a threshold.

\section{Protocols Based on Contended MAC}

\section{Core EXTRACTION Distributed Ad Hoc Routing}

The Core Extraction Distributed Ad Hoc Routing (CEDAR) algorithm was proposed in [41]. The basis for its name is the topology management, core extraction mechanism at the algorithm's heart. The core of a network is defined as the minimum dominating set (MDS), that is, all nodes are either part of this set or have a neighbor that is part of the set (see Fig. 6 ). The calculation of the MDS is a known NP-hard problem [41], hence the algorithm only finds an approximation of it. The reason for calculating the MDS, or the set of core nodes, is to provide a routing backbone. This ensures that every node can be reached, but not every node has to partake in route discovery. Non-core nodes save energy by not participating and this way protocol overhead is also reduced.
Furthermore, local broadcasts are highly unreliable due to the hidden and exposed node problems [41]. Within the core, reliable local unicasts may be used to propagate routing and QoS state information. This uses RTS-CTS handshaking to avoid hidden and exposed node problems and to make sure the "broadcast" packet is delivered to every neighboring core node. This scheme is termed core broadcast.

It was argued in [41] that using only local state for QoS routing incurs little overhead, but far from optimal routes may be computed, or in the worst case, no QoS route may be found, even if one exists. In the other extreme, gathering the whole network state at each node incurs a very high overhead, but in theory allows the computation of optimal paths, albeit with the possibility of using stale information. CEDAR compromises by keeping up-to-date information at each core node about its local topology, as well as the link-state information about relatively stable links with relatively high residual capacity further away.

This is done via increase and decrease waves. For every link, the nodes at either end are responsible for monitoring the available capacity on it and for notifying their dominators when it increases or decreases by a threshold value. The method of estimating available link capacity is not specified in [41]. In brief, increase and decrease waves carry notification by core broadcast of an increase or decrease in available capacity on a link, and the actual throughput achievable on it. They are processed such that increase waves travel slowly through the network but decrease waves travel quickly. This avoids the problem of nodes attempting to use link capacity that is no longer available. Any nodes receiving either type of message cache the relevant link capacity information. Increase waves have a time to live and are propagated as far as this allows. Decrease waves are only propagated further by nodes which had previous knowledge of the corresponding link, thus ensuring that the wave does not travel to parts of the network where it will be useless. If a link's capacity fluctuates, the fast-moving decrease wave quickly overtakes the slower increase wave and thus, information about unstable links is kept local. Highcapacity stable link information is allowed to propagate far.

When a source $s$ requires a route to destination $d$, with required throughput $b$, it must request this from its dominator, which will either know, or discover routes to the dominator of d using a core-broadcast search. This establishes so-called core paths.

When a QoS route is required, the shortest-widest core path satisfying the achievable throughput requirement is determined using a two-phase Dijkstra algorithm. However, nodes only have link capacity information from a limited radius due to the wave propagation mechanism. Thus, the QoS core path is determined in stages with each node routing as far as it can "see" capacity information, then delegating the rest of the routing to the furthest "seen" node on the core path. This process iterates until the final destination is reached and all links satisfy the achievable throughput requirement.

The greatest novelties of this technique were the core broadcast and link capacity dissemination mechanisms. These ensure efficient use of network resources and relatively accurate and up-to-date knowledge of the QoS state where it is required. Furthermore, this protocol does not rely on a TDMA network, as the protocols discussed in the previous section do. However, the problem of estimating available link capacities (achievable throughput) was left open.

\section{INTERFERENCE-AWARE QOS ROUTING}

In [43] the authors consider throughput-constrained QoS routing based on knowledge of the interference between links. 


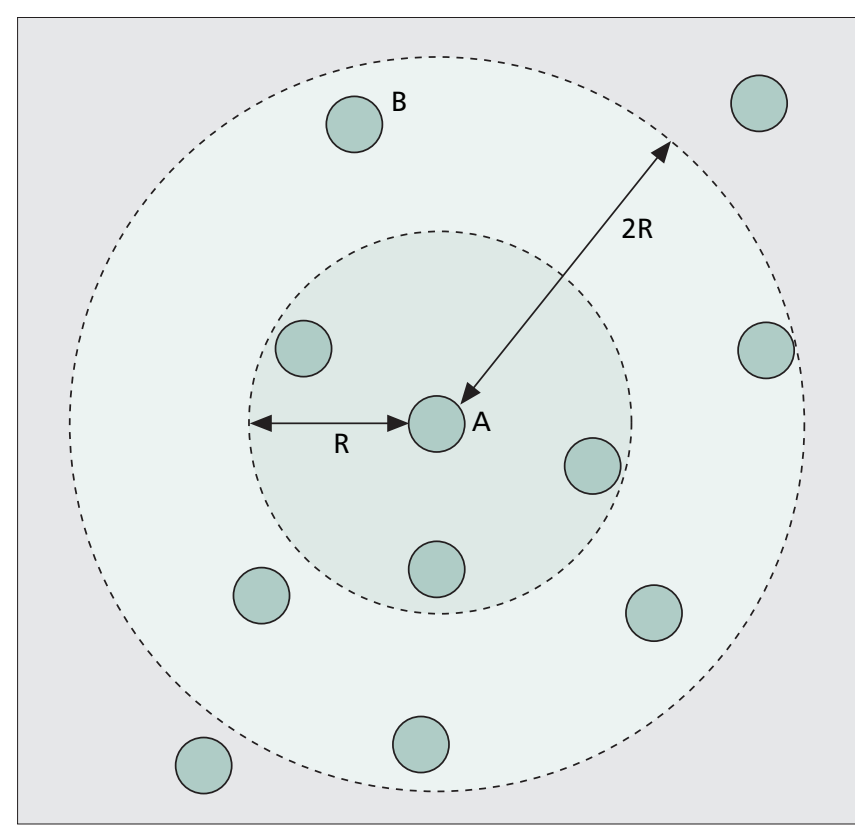

Figure 7. Illustration of node A's transmission range (circle radius $R$ ) and its carrier-sense range (circle radius $2 R$ ).

So-called clique graphs are established which reflect which links interfere with each other, thereby preventing simultaneous transmission. The proposed solution operates by first recording the channel usage $(\mathrm{b} / \mathrm{s})$ of each existing data session on each link. It is noted that the total channel usage of the sessions occupying the links within the same clique must not exceed the channel capacity. A link's residual capacity is then calculated by subtracting the channel usage of all sessions on links in the same clique from the link's nominal capacity. This link capacity information may then be used in any known distributed ad hoc routing protocol to solve the throughput-constrained routing problem.

Up until now, we have not discussed the heart of the problem of achievable throughput estimation in a contendedaccess network. This issue is the focus of work first presented in [12] and later published in [9].

A simple frequency reuse pattern is assumed, as shown in Fig. 7, wherein the carrier-sense range (cs-range) is twice the reception range. This means that if a node has a transmission range of $R$ meters, then any nodes at a distance of $\leq 2 R$ meters from it are within its carrier-sense range and vice versa. Nodes within the cs-range are termed cs-neighbors, and this set of nodes is the cs-neighborhood. The $c s$-range $=2 R$ model simulates the physical layer characteristics of network adapters which are able to sense the presence of a signal at a much greater range than that at which they are able to decode the information it carries.

In a contention-based MAC protocol such as the 802.11 distributed coordination function (DCF) [49], a node may only transmit when it senses the channel idle. Therefore, any nodes transmitting within its cs-range cause the channel to be busy and are thus in direct contention for channel access with it. This is one of the key realizations in $[9,12]$ : all nodes in the cs-range (cs-neighbors) must be considered when estimating a node's available channel capacity, that is, achievable throughput.

More specifically, in 802.11 the channel is deemed idle if both the transmit and receive states are idle and no node within $R$ has reserved the channel via the network allocation vector [12]. Knowing this, it is possible to statistically estimate a node's available channel capacity by measuring the fraction of time for which a node detects the channel state as idle.

A further major consideration in [12] is that nodes on a path carrying a data session interfere with each other as well. In the worst case, where the path is at least six nodes long, nodes in the middle of the path have two transmitters upstream and two downstream contending for the channel (due to the "cs-range $=2$-hops" model). This makes a total of five nodes in contention, that is, the contention count is five. For example, see Fig. 8, where a session requiring, say, $10 \mathrm{~kb} / \mathrm{s}$ is forwarded along the path $\{\mathrm{A}, \mathrm{B}, \mathrm{C}, \mathrm{D}, \mathrm{E}, \mathrm{F}\}$. Nodes $\mathrm{A}, \mathrm{B}$, $\mathrm{D}$, and $\mathrm{E}$ all must forward data at $10 \mathrm{~kb} / \mathrm{s}$ to satisfy the session's requirements. Therefore, at node $\mathrm{C}$, including its own channel usage, $50 \mathrm{~kb} / \mathrm{s}$ channel capacity is consumed. This is five times the session's nominal requirement, since the nodes are all contending for channel access with each other.

In $[9,12]$, the above considerations are used to extend an on-demand source-routing protocol to achieve throughputconstrained routing. Source routing is employed in order to be able to pin a data session to a particular route, unlike protocols such as AODV [50], which only store the next hop towards the destination at each node. Moreover, knowing the entire route length allows the maximum contention count to be easily calculated. However, since nodes share channel capacity with their cs-neighbors, each node must check that every single node in its cs-range has enough capacity to admit a session. To visualize this, see Fig. 8, where node G's csrange is shown to encompass nodes $\mathrm{B}, \mathrm{C}$, and $\mathrm{D}$. Therefore, $\mathrm{G}$ also falls in their cs-ranges. Continuing with the earlier example, each of these nodes is forwarding $10 \mathrm{~kb} / \mathrm{s}$, resulting in $30 \mathrm{~kb} / \mathrm{s}$ of channel capacity being consumed at node $\mathrm{G}$, even though it is not part of the route. To check that nodes such as $G$ can allow the session on path $\{A-G\}$ to be admitted, the cs-neighborhood of each node on the path is flooded with an admission request that carries the entire route the session would take. Each node receiving the admission request calculates the local capacity required by the session on the route. An "admission request denied" message is returned to the requesting node if the local capacity is not sufficient.

Another similar, yet also important, approach is proposed in [13], where the authors consider contention among csneighbors (nodes in each other's cs-ranges) in a similar way to [9]. The "cs-range $=2$ hops" model is adopted here also. However, instead of source routing, the contention-aware session admission mechanism is applied to AODV.

The algorithm for the residual channel capacity estimation relies on AODV's HELLO message mechanism. Each node records how many bits it inputs into the channel every second and it piggybacks this information on its periodic HELLO messages. Thus a node, say X, informs all of its neighbors of its channel usage. These neighbors propagate this information onto their neighbors (but only one hop) and thereby every node in X's cs-range learns its channel usage. Conversely, since all nodes implement this algorithm, $\mathrm{X}$ will know the channel usage of all of its cs-neighbors. All that remains to be done by $\mathrm{X}$ is to subtract the total channel usage of all these nodes from the raw channel capacity to obtain an estimate of the amount of free channel capacity that is available to it at that instant.

The major advantage of this protocol compared to the work in [9] is that no extra control packets are introduced, since bandwidth information is piggybacked on AODV's existing HELLO packets. However, one failing of this technique surfaces as illustrated in Fig. 7: consider node B which is inside the cs-range of node $\mathrm{A}$, but not inside the transmission radius of any of A's neighbors. Therefore, B cannot inform A of its channel usage, which therefore cannot be subtracted from A's available channel capacity.

While the approaches discussed in this section represent significant progress in achievable throughput estimation and 


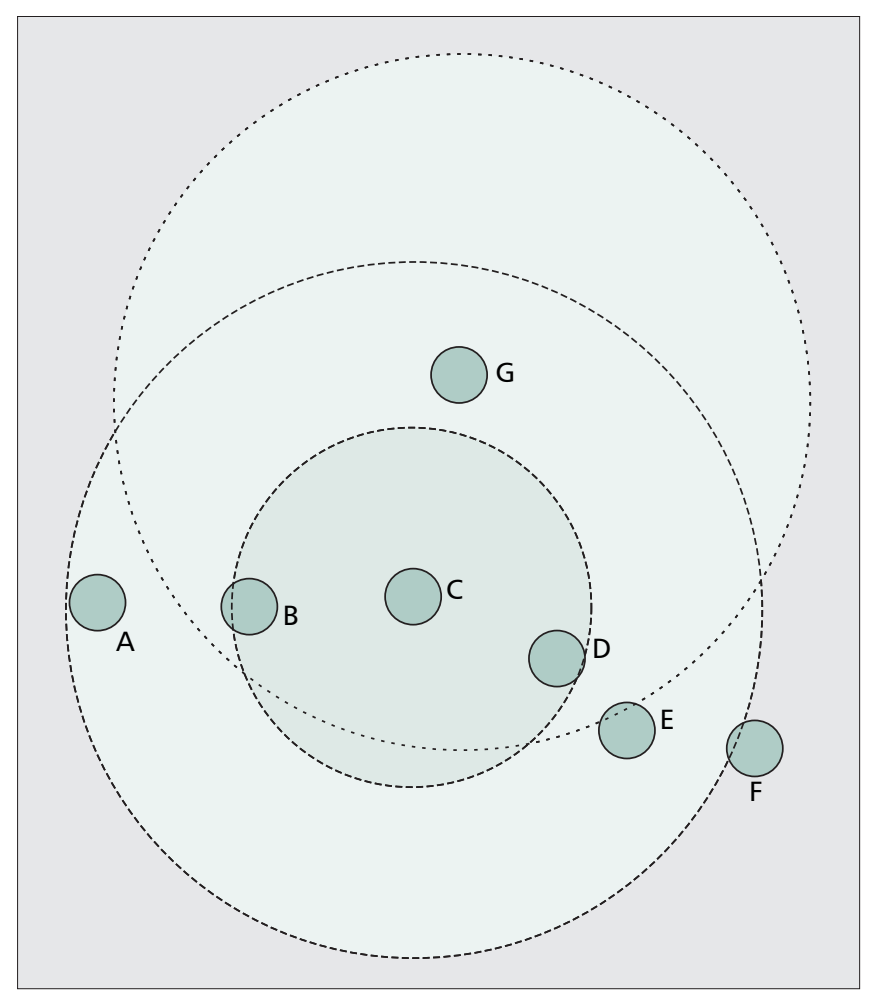

Figure 8. Illustration of mutual interference between nodes on a path $\{A-F\}$. The smaller and larger dashed circles represent node $C$ 's transmission and cs-ranges respectively and the large dotted circle is node G's cs-range.

admission control, and hence throughput-constrained QoS routing, there are still shortcomings. It is well known that as a network nears saturation, ready-to-send and data packet collisions (in a multihop network) become more frequent, wasting capacity. Additional capacity is wasted due to the 802.11 backoff algorithm, as the level of contention for the channel increases. The protocols discussed in this section do not consider these sources of wastage when calculating the residual capacity at each node. The need to include these factors has been recognized [51, 52]. In [52], we took a first step towards incorporating the effects of these factors in session admission control, employing approximate estimations of collision and back-off wastage in our QoS routing protocol.

\section{Cross-Layer Multiconstraint QOS Routing}

An approach proposed in [22] is the focus of this section. First of all, Fan proposes the MAC delay metric, which he defines as the time between a packet being received by the MAC protocol from the higher layers, and an ACK being received for it, after it is transmitted. This includes the time deferred when awaiting channel access and is thus a useful metric for avoiding busy links. Link reliability and throughput constraints are also considered in [22], but they use pre-existing definitions and methods of calculation.

The focus of the article is on performing multiconstraint QoS routing with the aforementioned three metrics. Fan reiterates the fact that the multiconstraint QoS routing problem is NP-complete [2] when a combination of additive and multiplicative metrics is considered. Among the above metrics, delay is additive, link reliability is multiplicative, and achievable throughput is concave. However, methods have been proposed (see [22] and references therein) for reducing this NP-complete problem to one that can be solved in polynomial time. In one such method, all QoS metrics, except one, take bounded integer values.
Then, the task of finding a path to satisfy all constraints can be performed by a modified Dijkstra's algorithm. In [22], the multiplicative metric is reduced to an additive one by taking the logarithm of the reliability percentage of a link. Also, the delay metric is reduced such that each link is represented by the percentage of the allowable total delay it introduces. The resulting problem in the new metric space can be solved in polynomial time. Then, a modified Bellman-Ford or Dijkstra's algorithm with the new reliability metric for link weights can be used to find an approximation to the optimal path. In each iteration, the total MAC delay along a path is checked and also paths which do not satisfy the channel capacity constraint are eliminated (see [22] for the exact algorithm used).

An obvious advantage of this approach is the concurrent consideration of several important QoS metrics in path selection. However, the QoS state for all paths must be discovered and kept fresh. This incurs extra overhead and the details of this mechanism are not discussed in [22]. Furthermore, as we have seen, such a protocol requires the presence of other mechanisms to actually measure the link reliability, MAC delay, and available channel capacity values at each node.

\section{ON-DEMAND DELAY-CONSTRAINED UnICAST ROUTING PROTOCOL}

A proposal in [35] focuses on providing delay-constrained routes for data sessions. The key features of this protocol are as follows. Firstly, a proactive distance vector algorithm is employed to establish and maintain routing tables containing the distance and next hop along the shortest path to each destination node. When a delay-constrained path is required, this information is used to send a probe to the destination along the shortest path to test its suitability. If this path satisfies the maximum delay constraint, the destination returns an ACK packet to the source, which reserves resources. For this purpose a resource reserving MAC protocol is assumed.

If the minimum hop path does not satisfy the delay constraint, the destination initiates a directed and limited flood search by broadcasting a RReq packet. Intermediate nodes forward the RReq if the total of their respective distances from the destination and source is below a set threshold and if the path delay is below the delay constraint value. When a copy of the RReq reaches the source with a path that meets the delay constraint, the route discovery process is complete.

While this protocol aims to minimize the hop distance between source and destination and discovers paths that satisfy a session's delay constraint, it has some major drawbacks. Firstly, while the aim of the directed flooding is to avoid global flooding, thereby reducing overhead compared to protocols based on that, extra overhead is incurred by the proactive distance-vector protocol which maintains the routing tables. Secondly, in [35] the existence of a resource reserving MAC is simply assumed. However, the authors do not discuss what kind of resources they wish to reserve and how this is to be achieved. Reserving channel capacity for example, is problematic, as previously discussed.

\section{QoS Greedy Perimeter Stateless Routing for ULTRA-WIDEBAND MANETS}

A recent proposal [18] at the time of this writing highlights a relatively new direction for MANETs: that of employing an ultra-wideband (UWB) physical layer. One of the advantages of UWB is that it allows a node's position to be estimated via triangulation techniques. This provides location information, without having to rely on GPS, for enabling a position-based routing protocol. The proposal in [18] extends an older proto- 
col, Greedy Perimeter Stateless Routing (GPSR) for QoS routing. We refer to this proposal as QGUM, meaning "QoS GPSR for UWB MANETs."

In brief, each node broadcasts beacons containing its ID and position to all of its neighbor nodes. The destination's position is learned at the same time as its ID. When a route is required, the source node sends a RReq to the neighbor node which is closest to the destination. The RReq specifies, among other information, the requesting data session's total delay bound, its PLR constraint, and the accumulated PLR so far.

A node receiving the RReq factors in its own PLR and compares the result with the PLR bound. If it is unacceptable, a Route Failure is sent back to the source node. In this case, the source node begins route discovery again, starting with a different node in its neighbor list. If the PLR bound is not exceeded, the intermediate node appends its ID to the RReq, in a manner akin to other source-routing protocols. It also adds its location before performing the same procedure as the source to find the next node to forward the RReq to. Each intermediate node performs the PLR checks and passes the RReq to the neighbor closest to the destination, until the destination receives the RReq.

The above procedure describes route discovery. We now summarize the method for ensuring QoS on routes. First of all, [18] suggests that QGUM can operate with either a contended MAC protocol, similar to the 802.11 DCF, or with a TDMA-based protocol such as 802.15.3 [46]. In the former case, available channel capacity is determined in the same way as in [9], described in the subsection "Interference-Aware QoS Routing" above, using channel idleness ratio estimation. In the latter, timeslots quantify channel capacity. However, as detailed above at the end of the subsection "QoS Routing in a CDMA over TDMA Network," we do not believe 802.15.3 is the ideal solution for multihop MANETs. Therefore, we focus on the contended MAC-based algorithm.

After a route to the destination is discovered as detailed above, the session admission control procedure begins. Owing to the available position information, the destination can calculate which nodes on the route are inside each other's csranges and thus which can transmit simultaneously. The destination then calculates the channel capacity required at each node for the data session to be admitted. It then sends an admission request (AdReq) back along the route. Each intermediate node checks its locally available capacity and the capacity of its cs-neighbors by flooding an AdReq, similar to the protocol in [9], as described above. If the intermediate node and all its cs-neighbors have sufficient capacity, they temporarily reserve the necessary capacity for the session and the AdReq is forwarded to the next hop in the route back towards the source node.

If any nodes or their cs-neighbors on the route have insufficient capacity, they generate an admission refused message. In essence this is passed to the next hop on the route towards the source, which invokes a path repair mechanism. This operates very similarly to the route discovery procedure, except only a partial new path must be discovered starting from the node before the one which had insufficient capacity. The main advantages of QGUM compared to earlier similar approaches described above are as follows:

- Exploitation of the multirate capability of the UWB physical layer

- Exploitation of the location information provided by the UWB physical layer, enabling directed route discovery

- Simultaneous satisfaction of an application's PLR and throughput requirements (delay can be considered instead of throughput)
However, these advantages must be balanced against the typically shorter range offered by UWB radios. For example, while UWB provides higher data rates than existing variants of $802.11 \mathrm{x}$, the approximate range for the proposed UWB $802.15 .3 \mathrm{a}$ specification is only $10 \mathrm{~m}$ at $110 \mathrm{Mb} / \mathrm{s}$ [53]. Indeed, current standardization efforts involving UWB radio technologies for wireless networks are targeted at personal area networks [54] and not larger-scale ad hoc WLANs as $802.11 \mathrm{x}$ is. This limits the applicability of protocols based on a UWB physical layer.

\section{PROTOCOLS INDEPENDENT OF THE TYPE OF MAC}

\section{QOS Optimized Link State Routing}

A QoS routing protocol based on Optimized Link State Routing (OLSR) [55] was presented in [44]. OLSR is a proactive protocol in which information about 1-hop and 2-hop neighbors is maintained in each node's routing table. This information is disseminated via periodically broadcast HELLO messages. OLSR minimizes the control overhead involved in flooding routing information by employing only a subset of nodes, termed multipoint relays (MPRs), to rebroadcast it. As a consequence, only MPRs are discovered during route discovery and thus only they are used as intermediate nodes on routes. Also, calculating the optimal MPR set to reach all 2hop neighbors is an NP-complete problem and therefore heuristics are applied.

Since only a subset of nodes are MPRs, the best links (as defined by some QoS metrics) may not be utilized for routing. In QoS-OLSR (QOLSR) [44], this problem is solved by proposing new heuristics for building nodes' MPR sets in order to enable QoS routing to take place. QOLSR employs both a variation on the MAC delay metric and the achievable throughput metric for QoS routing.

In contrast to many of the protocols discussed so far, although the analysis in [44] is based on the 802.11 MAC, QOLSR does not rely on the MAC protocol to provide residual channel capacity or delay information. These values are estimated statistically, using the periodic HELLO messages, as follows. The total expected MAC delay of a packet is a product of the average estimated delay or expected service time (EST) of one packet and the total number of packets awaiting transmission. The value of EST in turn depends on packets' transmission times and the expected number of retransmissions the MAC layer will have to perform (i.e., frame error ratio or FER). The FER is approximated by taking the ratio of the number of HELLO messages received during a monitoring window to the number expected, which is calculated from the known HELLO sending rate. The FER provides an estimate of the number of retransmissions required for successful delivery of a data packet.

The transmission delay of a packet depends on the amount of time a node spends backing off and resolving collisions. A detailed analysis in [44] shows that this is a function of the average backoff window size and the FER. Using these, the derived formulae yield an estimation for the EST of each packet and therefore the total MAC delay of a link between a node and its neighbor.

The achievable throughput of a link is also calculated statistically. The MAC delay or EST of a packet is estimated as described above. Using this, and knowledge of the overhead posed by packet headers and MAC control frames, the throughput experienced by packets can be estimated. 
To calculate the residual channel capacity on a link, the $\mathrm{MAC}$ protocol is required to notify the routing protocol when it transmits a packet. Queuing delay is estimated from the delay between passing a packet to the MAC protocol and receiving a "sent" notification, after subtracting the estimated time consumed by contention resolution and retransmissions. If there is no queuing delay, the queue is deemed empty. In this case, the elapsed time since the last notification was received, is considered the link's idle time. The total of this idle time as a fraction of the monitoring period is multiplied by the average throughput of a packet, to provide the estimate for residual channel capacity.

Finally, [44] details how nodes' MPR sets are constructed using the link capacity and delay information. It is claimed that the proposed heuristic selects the appropriate MPRs at each node in order to ensure that nodes are connected via the highest residual capacity and lowest delay paths.

In summary, QOLSR appears to be a promising proactive QoS routing protocol for finding and maintaining the shortest-widest paths in terms of delay and throughput. It also benefits from the characteristic lower overhead (compared to earlier proactive protocols) of OLSR, due to the use of MPRs. While QOLSR does not rely on the use of lower layer information directly, it does require notifications to be sent by the MAC protocol in order to calculate QoS metrics. Avoiding complicated MAC-routing interactions is a bonus, but the achievable QoS estimations are inherently not as accurate as with MAC layer idle-time estimation.

\section{Link Stability-BASED Routing}

In [21], link stability is considered as an important QoS metric. Stability is defined as the expected lifetime of a link, which is largely dependent on the node movement pattern [21]. The article presents the probability distribution functions (PDF) of link lifetimes under various node movement models. The remaining link lifetime is estimated as the area under the PDF for a given mobility model, taken between the link's measured lifetime so far, and infinity. For example, in the random destination mobility model, nodes do not change direction after selecting a destination, until they reach it. This mobility model was found to produce a link lifetime PDF similar to a Rayleigh distribution [21]. To find the probability that a link's remaining lifetime is greater than a time $t$, the PDF of the link lifetime is integrated between $t+L_{p}$ and infinity, where $L_{p}$ is the link's past lifetime.

A link lifetime model such as the one above is proposed for each of a selection of mobility models. An application may specify a lower limit for acceptable path failure probability, $P_{\text {fail }}$. This value can be calculated based on a data session's delay, delay jitter, and packet loss rate requirements.

It was proposed [21] that this mechanism be combined with AODV for QoS routing. The value $P_{\text {fail }}$ is inserted into RReq packets. Intermediate nodes test that the cumulative failure probability of links up to that point (also stored in the RReq and updated by each node) is not greater than $P_{\text {fail }}$ : Therefore, using an appropriate model such as the above and given the data session's duration, it is possible to calculate the probability of a path remaining intact for the duration of the data session, $P_{\text {survive }}$. If this is unacceptable, that is, $P_{\text {survive }}<$ $P_{\text {fail }}$, then the session is not admitted.

This simple mechanism could be useful for statistically predicting link lifetimes and therefore avoiding links and paths that have a high probability of failure while a session is active. An obvious difficulty with this approach is that the node mobility pattern must be known and must be modeled accurately for the lifetime estimation to be useful. However, com- bined with other stability metrics, as discussed below, this could be a useful component of a more sophisticated QoS provisioning mechanism.

Another approach that considers link and path stability as an important QoS metric is presented in [42]. A new variation on the stability metric is introduced in the form of the entropy metric. This is defined for a link as a function of the relative positions and velocities, and the transmission ranges of the link's two end nodes. A path's entropy is defined as the product of the link entropies along it. The lower the entropy, the higher the path stability.

This scheme is incorporated into a source-routed scheme somewhat akin to DSR, and during route discovery, the path entropy (among other metrics) is calculated. A destination receives RReqs over multiple paths and waits a specified interval after receiving the first one, before selecting the path with the lowest entropy, that is, highest stability. This route is returned to the source in the RRep, thereby completing the route discovery.

This approach has the potential to be more accurate than that in [21], since it considers nodes' relative positions and velocities for calculating the probability of link failure, rather than just a general PDF for a given mobility model. However, this comes at the price of assuming that each node is capable of determining its position via GPS or some similar system [42].

\section{Hybrid Ad Hoc Routing Protocol}

The Hybrid Ad hoc Routing Protocol (HARP) is introduced in [25]. It uses the notion of quality of connectivity (QoC) as its routing metric. This is defined as a function of two node states: residual buffer space and relative stability. The latter is defined for node $x$ over a chosen period of time, $t_{1}-t_{0}$, as:

$$
\operatorname{stab}(x)=\left|\frac{N_{t_{0}} \cap N_{t_{1}}}{N_{t_{0}} \cup N_{t_{1}}}\right|
$$

where $N_{t_{0}}$ and $N_{t_{1}}$ are the set of neighbors of $x$ at times $t_{0}$ and $t_{1}$, respectively. Thus, stability is greater, the fewer the number of neighbor nodes that change between $t_{0}$ and $t_{1}$. The higher a node's residual buffer space and relative stability, the better the $\mathrm{QoC}$ to it is.

The QoC of each node is used in a logical topology construction algorithm. Each node periodically broadcasts a beacon to all of its neighbors, which contains its address and QoC. Then, each node selects as its preferred neighbor (PN) the neighbor node with the highest QoC. A link between a node and its PN is called a preferred link. A logical tree is constructed by connecting nodes together using only preferred links. A tree's growth terminates where a node's preferred link is with a node that is already part of the tree. This heuristic has been proven to yield a forest of trees [25]. In brief, each tree is then considered a routing zone, within which proactive routing occurs. Interzone routing is performed on-demand, and hence the hybrid route discovery of this protocol.

In interzone routing, other zones may be abstracted as nodes, thus a packet can be routed to another zone, and on arrival, the intrazone routing mechanism can direct the packet to its final destination.

HARP also includes route discovery optimizations which reduce overhead. Firstly, the forest structure can be used to avoid having to flood route request (RReq) packets used in interzone routing. This is done by forwarding RReqs only via gateway nodes; a node is considered to be a gateway, if it is the neighbor of a leaf node, but it is in another zone. 
Secondly, features of the relative distance micro-discovery routing protocol (RDMAR) [56] are incorporated into HARP. RDMAR does not limit the number of neighbors propagating a flooded packet, but limits the scope of the flooding instead. Thus, RReqs do not propagate to areas of the network where they will be useless, thereby wasting resources.

The time-to-live (TTL) field in a RReq is set based on an estimation of the relative distance of the destination in terms of hops. However, the estimation can only be made if there is some previous knowledge of the destination, and a replacement path to it is sought, that is, this is not the first search. In this case, the relative stabilities of each node on the path, combined with the time elapsed since the stabilities were recorded, yields an estimation for the total maximum change in the positions of the nodes on the path. This is added to the previous known distance in meters (hops $\times$ radio range) of the destination. The sum is divided by the radio range to obtain an estimated upper bound on the distance of the destination in number of hops. This value is used for the TTL.

A further enhancement to RDMAR in HARP, is that intermediate nodes may make their own estimation for the distance to the destination. If this is higher than the original estimation, it implies that the destination does not lie in this direction from the source. In this case, the RReq is not propagated further, meaning that it does not travel to areas of the network where the destination surely does not lie.

HARP's use of the QoC metric allows it to discover routes that have fewer buffered packets and which are relatively stable. This results in lower average delay and fewer mid-session route failures, potentially yielding a lower session dropping rate. Additionally, QoC-based routing produces a load-balancing effect, which avoids congestion and early battery drainage of any single node, thereby delaying network partitioning.

On the downside, HARP does not consider an application's particular requirements, it aims only to improve average packet delay and network lifetime and to reduce the chance of route failure during a data session. Moreover, the beaconing process results in higher routing overhead compared to purely reactive protocols such as DSR.

\section{Delay-Sensitive Adaptive Routing Protocol}

The Delay-Sensitive Adaptive Routing Protocol (DSARP) [19] employs reactive route discovery, is completely decoupled from the MAC protocol, and provides delay guarantees for time-sensitive data sessions. Its basic operation is very similar to classical reactive MANET routing protocols such as DSR. However, when a path is required for delay-sensitive traffic, a different algorithm is employed.

The source node sends a route request (RReq), as usual. This is allowed to propagate to the destination, which sends a route reply (RRep). When forwarding the RRep, each intermediate node on the path attaches the number of packets awaiting transmission in its buffer. Multiple RReps may be received by the source node, which then selects several shortest paths, if there are multiple. Alternatively, the shortest path plus the next shortest path are selected. Using the information about buffer usage at each node, the source calculates the total number of packets on each selected path. Finally, the traffic flow on each path is adjusted such that the new traffic allocated to it is greater if the existing traffic on it is lower and the number of packets on other paths is greater. This algorithm pushes the network towards a state where each path has an equal flow of traffic on it and thus is likely to produce the same packet delay. Essentially, this implements a form of loadbalancing, ensuring that the energy usage of nodes is also distributed evenly. After adjusting the traffic on each path, a statistical guarantee can be made about the delay on that path.

DSARP is simple to implement and provides delay guarantees without relying on the MAC protocol, but has the following disadvantages. The number of buffered packets on each path must be rediscovered each time a new session begins, regardless of whether the route has failed or not. This incurs extra overhead. Also, the delay guarantees may fail in the face of mobility, if other nodes move into contention range and cause greater channel access delays for nodes on a session's path.

\section{APPLICATION-AWARE QOS ROUTING}

A rather unique approach to QoS routing is presented in [17]. It is unique because instead of using lower layer (MAC) information, it is based on the aid of the transport layer. The proposal, referred to as Application Aware QoS Routing (AAQR) in the literature, assumes the use of the real-time transport protocol (RTP) [57]. The delay between two nodes is estimated statistically by examining the difference between time stamps on transmission and receipt of RTP packets between those two nodes. The delay variance is also calculated. Furthermore, each node records the throughput requirement of RTP sessions which are flowing through it. Subtracting the total of these throughput values from the raw channel capacity gives an estimate for the total remaining capacity at that node.

When a QoS-route is required, applications may specify throughput and delay constraints. In [17] delay is considered the most important constraint for multimedia applications. Routes are discovered on-demand, although the details of the route-discovery procedure are not discussed. A subset of the discovered routes is selected, such that all paths satisfy the delay constraint of the application. From this subset a further subset of routes is selected, which also satisfy the application's throughput constraint. Finally, from this second subset, the route with the lowest variance in RTP packet transmission delays, is chosen. If there are no routes that meet the throughput requirement, the route with the highest available channel capacity, which satisfies the delay constraint, is selected.

A major advantage of AAQR is that no extra overhead is incurred for QoS routing, since the existing transport layer packets are used for QoS metric estimation. Additionally, both delay and throughput constraints may be considered. However, the use of RTP is assumed, and therefore the range of application scenarios for this protocol is obviously limited.

\section{Genetic Algorithm-Based QoS Routing}

In [24], a Genetic Algorithm-based source-routing protocol for MANETs (GAMAN) is proposed, which uses end-to-end delay and transmission success ratio for QoS metrics. Genetic algorithms (GAs) may be employed for heuristically approximating an optimal solution to a problem, in this case finding the optimal route based on the two QoS constraints mentioned.

The first stage of the process involves encoding routes so that a GA can be applied; this is termed gene coding. For this purpose, paths are discovered on-demand and then a network topology view is constructed in a logical tree-like structure. Each node stores a tree routed at itself with its neighbor nodes as child nodes and in turn their neighbor nodes as their children. Tree reductions are used to avoid duplicate subtrees (see [24]). Each tree junction is considered a gene and multiple genes make up a chromosome which represents a path.

The route discovery algorithm is assumed to collect locally computed metrics such as average delay over a link and the link reliability for the links on each path. After the gene encoding 
stage, the fitness $T$ of each path is calculated as follows:

$$
T=\frac{\sum_{i=1}^{n} D_{i}}{\prod_{i=1}^{n} R_{i}}
$$

where $D_{i}$ and $R_{i}$ are the delay and reliability of link $i$, respectably. The fitness values are used to select paths for cross-over breeding and mutation operations. The fittest path (with the smallest $T$ ) and the offspring from the genetic operations are carried forward into the next generation.

While this method is a useful heuristic for approximating the optimal value over the delay and link reliability metrics at the same time, it requires many paths to be searched in order to collect enough "genetic information" for the GA operations to be meaningful. This means that the method is not suited to large networks, as the authors themselves admit [24]. The methods of calculating $D_{i}$ and $R_{i}$ are not detailed, but we assume they can be calculated statistically by the end nodes of each link.

Collecting and maintaining sufficient route and QoS state information in order to make a GA useful for QoS routing is costly in terms of both overhead and energy consumption. However, heuristic methods are often the only feasible way of solving NP-complete multiconstraint multihop QoS routing problems. Thus, while their general applicability to MANETs is limited, GAs may play a niche role in finding near-optimal routes, while satisfying multiple QoS constraints in certain environments. For example, MANETs which are less powerconstrained and experience lower levels of mobility, and/or MANETs having topologies where a relatively small number of nodes can be combined in a relatively large number of ways to construct valid routes. The GAMAN protocol discussed in this section provides an exploratory example of how GAs may possibly be applied in such networks.

\section{ENERGY- AND RELIABILITY-AWARE ROUTING}

The Maximum Residual Packet Capacity (MRPC) protocol is proposed in [23], which considers battery charge as well as link reliability during route selection. Admittedly, MRPC is not intended to be a QoS routing protocol, but we consider it here since it utilizes some QoS-related metrics to improve allround QoS. Routing based on residual battery charge is considered extensively in the literature [33]. However, in our view, protocols that consider only this state are not useful for QoS routing, since they do not improve the QoS experienced by individual data sessions or packets. On the other hand, MRPC also considers link reliability, as detailed below.

In [23] a node-link metric is introduced to capture the energy lifetime of a link between nodes $i$ (transmitter) and $j$, which is defined as:

$$
L_{i, j}=\frac{R_{i}}{E_{i, j}}
$$

where $R_{i}$ is the residual battery charge at node $\mathrm{i}$ and $E_{i, j}$ is the energy required to transmit a data packet of a given size over the link $(i, j)$. A suggested formulation for $E_{i, j}$ is as follows:

$$
E_{i, j}=\frac{T_{i, j}}{\left(1-p_{i, j}\right)^{H}}
$$

where $T_{i, j}$ is the energy required for one transmission attempt of the aforementioned data packet with a fixed transmission power. Also, $p_{i, j}$ is the packet error probability of the link $(i, j)$ and $H=1$ if hop-by-hop retransmissions are performed by the link layer. From the above formulas, it is clear that the lifetime of a link is higher when greater battery charge remains at the transmitter node and when the reliability of the link is high, resulting in a low energy cost for correctly transmitting a packet. These formulas give an estimation for the expected number of data packets that can be transmitted over a link before the battery of the transmitter fails [23]. Then, if a route failure is said to occur when any single link on it fails, the lifetime of path $p$ in number of packets is simply:

$$
\text { Life }_{p}=\min _{(i, j) \in p}\left\{L_{i, j}\right\}
$$

MRPC considers the best route to be the one with the greatest residual lifetime. In [23], it was suggested that the MRPC algorithm be implemented in AODV [50] for application in MANETs. As routes are discovered, the lifetime of the path is accumulated by calculating the lifetime of each link. The next hop to a destination is always selected to be the neighbor which results in the greatest possible value for Life $e_{p}$.

This protocol results not only in load balancing, increasing the life of the network and avoiding congestion, but also yields closer-to-optimal energy consumption per packet, as well as lower packet delay and packet loss probability, due to the preference for more reliable links. It can also be implemented in an on-demand fully distributed routing protocol, such as AODV. However, link reliabilities must somehow be estimated, which may nsot be a trivial problem. Furthermore, like HARP, MRPC does not cater to particular sessions' requirements, only fosters better all-round QoS, and hence may be unsuitable for many applications. On the other hand, as mentioned above, MRPC is not primarily intended to be a QoS routing protocol, rather an energy-efficient best effort protocol.

\section{TrendS AND Progress IN THE FIELD}

As discussed above, many of the earlier QoS routing proposals (pre-2000) for MANETs were based on contention-free MAC protocols and relied on either TDMA or TDMA/CDMA channel access mechanisms. This was probably due to their well-understood nature from the field of cellular communications. A TDMA approach offers a straightforward method of quantifying channel capacity and access opportunities, as well as allowing such opportunities to be deterministically reserved for particular application data sessions. This enables throughput guarantees to be made, provided that the network dynamics do not invalidate them. Due to mobility, as well as the unpredictable nature of the wireless channel, truly hard guarantees can never be made in a MANET.

Even though some newer proposals continue to assume TDMA, we (and others [9]) believe that nonhierarchical TDMA-based methods are practically highly unfeasible in MANETs, since time-slotting requires global clock synchronization, which is difficult to achieve in a mobile environment. A further drawback of this approach is the high signaling overhead incurred by slot scheduling and the potential complexities thereof [40].

Newer MAC protocols such as that specified by 802.15 .3 [46] offer feasible TDMA solutions for MANETs by introducing node hierarchies whereby a group of nodes in a piconet is synchronized by a central controller node. However, this protocol is designed only for personal area networks and not for large-scale multihop MANETs. On the other hand, CDMAbased methods introduce the problem of code allocation in a 
dynamic mobile environment. In light of these conclusions, we believe, as previously stated, that QoS routing methods that rely on such channel access methods are not ideal for general, and especially larger-scale MANETs.

This is reflected in the literature, since the majority of later solutions (post-2000), are based on contended MAC protocols (generally 802.11) or do not rely on any set channel access mechanism to be in place. Previously, we discussed several proposals relying on a contended MAC protocol, such as 802.11. Many less mature solutions in this category did not consider the nature of contention between neighboring nodes sufficiently accurately and thus reliable QoS provisioning did not become a reality for MANETs. It was through key works such as $[9,13]$, that the nature of contention and its effect on (primarily throughput-constrained) QoS routing, begun to be well-understood. Other newer proposals take this understanding as a basis for further QoS routing designs. Proposals such as those discussed earlier greatly further the field of QoS session admission control. This was one of the areas identified as future work in previous surveys discussed above.

Many solutions continue to be based upon 802.11x and its CSMA/CA-based channel access mechanism. Even though 802.11 is an aging standard, the CSMA/CA mechanism has survived into its most recent versions and therefore proposals based on the 802.11 MAC protocol continue to be very relevant. On the other hand, QoS routing proposals based on an ultra-wideband physical layer (for example, [18]) are emerging. As we discussed above though, UWB radios have a limiting shorter range compared to 802.11x. Accordingly, current UWB standardization efforts are all aimed at personal area networks, meaning that UWB-based QoS routing proposals have limited applicability to small-scale MANETs only.

Statistical QoS Protocols that make no assumptions about the MAC layer have also received greater attention in the last five years. Such protocols allow a simpler modular network stack design, without the complications of cross-layer issues. However, no guaranteed level of service is provided, as we saw in the proposals discussed above. Instead, such protocols generally improve the all-around average QoS experienced by packets under some metrics, at the expense of other performance metrics or increased complexity or overhead. Such protocols may not be sufficient for supporting applications with stringent QoS requirements. By contrast, protocols in this category have done much to improve QoS robustness to failures, which was another area identified as future work in previous surveys. The link and node stability-based techniques that were summarized earlier can find longer-lasting routes and thus improve the robustness of QoS solutions against failures caused by mobility.

In summary, we can say that there is a trend for QoS routing solutions to move away from contention-free MAC dependence and towards contended-MAC dependence for throughput-constrained applications. To cater for many other metrics, such as delay and PLR, numerous statistical protocols which are independent of the MAC layer, have been proposed.

Another aspect of development considers the metrics themselves. Again, in the earlier proposals, the focus was on providing an assured throughput service only, since throughput was deemed the most important requirement. Some earlier protocols could serve, for example, either a throughput or a delay requirement, but not both simultaneously. In this context, the trend we observe has been to move from single-constraint routing to multiconstraint routing, as demonstrated by the later proposals we have discussed. However, multiconstraint routing remains an NP-complete problem $([2,48])$ and thus most of the described solutions do not aim to find opti- mal routes. Instead, they simply apply multiple metrics to route filtering, removing all that do not satisfy a particular constraint. One exception was described, in which a genetic algorithm is employed as an heuristic to finding the optimal route based on more than one metric.

\section{FUTURE WORK}

Following the work summarized above, we believe that there is still a way to go in the area of throughput-constrained routing, before perfect SAC is achieved, even in a low-mobility scenario. Works such as $[9,13]$ consider channel contention, as well as MAC overheads in achievable throughput estimation, but the time wasted due to deferring transmission, random back-off, and collisions has not been considered. The wastage due to collisions is especially difficult to calculate in a multihop environment. This is important future work, if accurate residual channel capacity estimation is to be realized with contented MAC. The understanding of contention among nodes also needs to be transferred to considerations of other QoS metrics, such as end-to-end packet delay, which is affected by the queues of all nodes within contention range [34]. Delay jitter and energy consumption (due to collisions), are also affected. Quantifying the impact on these metrics and more, in the light of contention awareness and collisions, designing routing protocols that incorporate this knowledge and evaluating them with realistic application layer models, is all future work.

A further trend that we have observed is that many designers place great emphasis on the session admission (QoS route finding) capability of their protocol, which is admittedly very important. In contrast, they often neglect or downplay the importance of session completion, that is, maintaining the routes and the QoS for as long as an application data session requires. An aspect of this, QoS robustness, was highlighted by earlier survey writers. However, more work on the evaluation of QoS-sensitive session completion performance with realistic application layers, would be useful. Ultimately, session completion is more important from a user perspective, than session admission. This is because the perceived QoS is better when some sessions are blocked but none are dropped mid-session, rather than all sessions being admitted, but some failing. Furthermore, fast local QoS route-repairing schemes require additional investigation to improve QoS session completion rates and protocols' robustness against mobility.

Previously we reiterated that one of the major challenges to the provision of QoS in MANETS is the unreliable wireless channel. However, we have found that the majority of QoS routing protocol evaluation studies assume a perfect physical channel, ignoring the effects of shadowing and multipath fading. Therefore, studying the impact of a more realistic physical layer model on QoS routing protocol performance is another interesting area of future work.

As mentioned in the previous section, while simple multiconstraint QoS routing proposals are numerous, there are few that attempt to optimize multiconstraint routing. One example was based on genetic algorithms [24]. However, such methods have limited applicability due to the overhead and energy cost of collecting enough state information. Accurate studies are required to establish, with various networking environments and topologies, whether or not it is feasible to collect and maintain sufficient state information to apply methods such as GAs. For the cases where it is, more research is required on different types of heuristic algorithms for calculating nearoptimal paths with multiple QoS constraints. Comparative studies on the performance and impact of the heuristics, are 
additional future work. Moreover, there is a distinct lack of protocol frameworks for incorporating such methods into practically realizable systems. One promising, but perhaps not yet mature or feasible, approach is that of Node State Routing [34], which we discussed above. Such a solution would provide the mechanism by which to disseminate the information to enable multiconstraint QoS routing.

\section{SUMMARY}

In this article we reviewed the challenges to and basic concepts behind QoS routing in MANETs and provided a thorough overview of QoS routing metrics and design considerations. We then classified many of the major contributions to the QoS routing solutions pool published in the period 1997-2006. The protocols were selected in such a way as to highlight many different approaches to QoS routing in MANETs, while simultaneously covering most of the important advances in the field since the last such survey was published. We summarized the operation, strengths, and drawbacks of these protocols in order to enunciate the variety of approaches proposed and to expose the trends in designers thinking. The protocols' interactions with the MAC layer were also described. Finally, we provided an overview of the areas and trends of progress in the field and identified topics for future research.

\section{REFERENCES}

[1] D. Kim, "A New Mobile Environment: Mobile Ad Hoc Networks (MANET)," IEEE Vehic. Tech. Soc. News, Aug. 2003, pp. 29-35.

[2] S. Chen, Routing Support for Providing Guaranteed End-toEnd Quality-of-Service, Ph.D. thesis, University of Illionois at Urbana-Champaign, 1999

[3] S. Chakrabarti and A. Mishra, "QoS Issues in Ad Hoc Wireless Networks," IEEE Commun. Mag., vol. 39, Feb. 2001, pp. $142-48$.

[4] S. Chakrabarti and A. Mishra, "Quality of Service Challenges for Wireless Mobile Ad Hoc Networks," Wiley J. Wireless Commun. and Mobile Comp., vol. 4, pp. 129-53, Mar 2004.

[5] J. N. Al-Karaki and A. E. Kamal, "Quality of Service Routing in Mobile Ad Hoc Networks: Current and Future Trends," Mobile Computing Handbook, I. Mahgoub and M. Ilays, Eds., CRC Publishers, 2004.

[6] T. B. Reddy et al., "Quality of Service Provisioning in Ad Hoc Wireless Networks: A Survey of Issues and Solutions," April 2004, available online: http://www.sciencedirect.com

[7] S. Saunders, Antennas and Propagation for Wireless Communication Systems Concept and Design, New York: Wiley, 1999.

[8] C. E. Perkins, Ed., Ad Hoc Networking, Ch. 3., Addison Wesley, 2001.

[9] Y. Yang and R. Kravets, "Contention-Aware Admission Control for Ad Hoc Networks," IEEE Trans. Mobile Comp., vol. 4, Aug 2005, pp. 363-77.

[10] L. Kleinrock and F. Tobagi, "Packet Switching in Radio Channels, Part II: The Hidden Terminal Problem in Carrier Sense Multiple-Access Modes and the Busy-Tone Solution," IEEE Trans. Commun., vol. 23, no. 12, 1975, pp. 1417-33.

[11] D. Shukla, L. Chandran-Wadia, and S. Iyer, "Mitigating the Exposed Node Problem in IEEE 802.11 Ad Hoc Networks," Proc. 12th Int'l. Conf. Computer Commun. and Networks, Oct. 2003, pp. 157-62.

[12] Y. Yang and R. Kravets, "Contention-Aware Admission Control for Ad Hoc Networks," tech. rep., University of Illinois at Urbana Champaign, 2003.

[13] L. Chen and W. Heinzelman, "QoS-Aware Routing Based on Bandwidth Estimation for Mobile Ad Hoc Networks," IEEE JSAC, vol. 23, Mar. 2005, pp. 561-72.

[14] C. R. Lin and J.-S. Liu, "QoS Routing in Ad Hoc Wireless Networks," IEEE JSAC, vol. 17, Aug. 1999, pp. 1426-38.

[15] S. Chen and K. Nahrstedt, "Distributed Quality-of-Service
Routing in Ad Hoc Networks," IEEE JSAC, vol. 17, Aug. 1999, pp. $1488-505$.

[16] A. R. Bashandy, E. K. P. Chong, and A. Ghafoor, "Generalized Quality-of-Service Routing with Resource Allocation," IEEE JSAC, vol. 23, Feb. 2005, pp. 450-63.

[17] M. Wang and G.-S. Kuo, "An Application-Aware QoS Routing Scheme with Improved Stability for Multimedia Applications in Mobile Ad Hoc Networks," Proc. IEEE Vehic. Tech. Conf., Sept. 2005, pp. 1901-05.

[18] A. Abdrabou and W. Zhuang, "A Position-Based QoS Routing Scheme for UWB Mobile Ad Hoc Networks," IEEE JSAC, vol. 24, Apr. 2006, pp. 850-56.

[19] M. Sheng, J. Li, and Y. Shi, "Routing Protocol with QoS Guarantees for Ad-Hoc Network," Electronics Letters, vol. 39, Jan. 2003, pp. 143-45.

[20] S. Singh, M. Woo, and C. S. Raghavendra, "Power-Aware Routing in Mobile Ad Hoc Networks," Proc. Int'l. Conf. Mobile Computing and Networking, 1998, pp. 181-90.

[21] I. Rubin and Y.-C. Liu, "Link Stability Models for QoS Ad Hoc Routing Algorithms," Proc. 58th IEEE Vehic. Tech. Conf., vol. 5, Oct. 2003, pp. 3084-88.

[22] Z. Fan, "QoS Routing Using Lower Layer Information in Ad Hoc Networks," Proc. Pers., Indoor and Mobile Radio Commun. Conf., Sep. 2004, pp. 135-39.

[23] A. Misra and S. Banerjee, "MRPC: Maximising Network Lifetime for Reliable Routing in Wireless Environments," Proc. IEEE Wireless Commun. and Networking Conf., Orlando, FL, March 2002.

[24] L. Barolli, A. Koyama, and N. Shiratori, "A QoS Routing Method for Ad Hoc Networks Based on Genetic Algorithm," Proc. 14th Int'l Wksp. Database and Expert Systems Applications, Sept. 2003, pp. 175-79.

[25] N. Nikaein, C. Bonnet, and N. Nikaein, "Hybrid Ad Hoc Routing Protocol - HARP," Proc. Int'l. Symp. Telecommun., 2001.

[26] D. Kim, C.-H. Min, and S. Kim, "On-Demand SIR and Bandwidth-Guaranteed Routing with Transmit Power Assignment in Ad Hoc Mobile Networks," IEEE Trans. Veh. Tech., vol. 53, pp. 1215-23, July 2004.

[27] N. Wisitpongphan et al., "QoS Provisioning using BER-Based Routing in Ad Hoc Wireless Networks," Proc. Vehic. Tech. Conf., vol. 4, May 2005, pp. 2483-87.

[28] C.-K. Toh, "Maximum Battery Life Routing to Support Ubiquitous Mobile Computing in Wireless Ad Hoc Networks," IEEE Trans. Commun., vol. 39, no. 6, 2001, pp. 138-47.

[29] C. E. Perkins et al., "Performance Comparison of Two OnDemand Routing Protocols for Ad Hoc Networks," IEEE Pers. Commun. Mag., vol. 8, Feb. 2001, pp. 16-28.

[30] J. Broch et al., "A Performance Comparison of Multi-Hop Wireless Ad Hoc Network Routing Protocols," Proc. Int'I. Conf. Mobile Computing and Networking, Oct. 1998.

[31] J.-H. Chang and L. Tassiulas, "Energy-Conserving Routing in Wireless Ad-Hoc Networks," Proc. IEEE INFOCOM, vol. 1, 2000, pp. 22-31.

[32] S. Doshi, S. Bhandare, and T. Brown, "An On-Demand Minimum Energy Routing Protocol for a Wireless Ad-Hoc Network," Mobile Computing and Commun. Rev., vol. 6, no. 2, 2002, pp. 50-66

[33] C. Yu, B. Lee, and H.-Y. Youn, "Energy-Efficient Routing Protocols for Mobile Ad-Hoc networks," Wiley J. Wireless Commun. and Mobile Comput., Dec. 2003, pp. 959-73.

[34] J. Stine and G. de Veciana, "A Paradigm for Quality of Service in Wireless Ad Hoc Networks using Synchronous Signalling and Node States," IEEE JSAC, vol. 22, Sept. 2004, pp. 1301-21.

[35] B. Zhang and H. T. Mouftah, "QoS Routing for Wireless Ad Hoc Networks: Problems, Algorithms, and Protocols," IEEE Commun. Mag., vol. 43, Oct. 2005, pp. 110-17.

[36] M. Grossglauser and D. Tse, "Mobility Increases the Capacity of Ad Hoc Wireless Networks," IEEE/ACM Trans. Net., 2002.

[37] E. Neely and M.J. Modiano, "Capacity and Delay Trade-Offs for Ad Hoc Mobile Networks," IEEE Trans. Info. Theory, 2005.

[38] L. Galluccio and S. Morabito, and G. Palazzo, "Analytical Evaluation of a Trade-Off Between Energy Efficiency and Responsiveness of Neighbor Discovery in Self-Organizing Ad Hoc Network," IEEE JSAC, vol. 22, Sept. 2004, pp. 1167-82.

[39] D. Haenggi, and M. Puccinelli, "Routing in Ad Hoc Networks: 
A Case for Long Hops," IEEE Commun. Mag., vol. 43, Oct. 2005, pp. 93-101.

[40] T.-W. Chen, J. T. Tsai, and M. Gerta, "QoS Routing Performance in Multihop, Multimedia, Wireless Networks," Proc. IEEE 6th Int'l. Conf. Universal Personal Communications, vol. 2, Oct 1997, pp. 557-61.

[41] R. Sivakumar, P. Sinha, and V. Bharghavan, "CEDAR: A CoreExtraction Distributed Ad Hoc Routing Algorithm," IEEE JSAC, vol. 17, Aug. 1999, pp. 1454-65.

[42] H. Shen, et al., "A Distributed Entropy-Based Long-Life QoS Routing Algorithm in Ad Hoc Network," Proc. IEEE Canadian Conf. Electrical and Computer Eng., vol. 3, May 2003, pp. 1535-38.

[43] R. Gupta et al., "Interference-Aware QoS Routing (IQRouting) for Ad-Hoc Networks," Proc. Global Telecommun. Conf., vol. 5 , pp. 2599-604, Nov. 2005.

[44] H. Badis and K. A. Agha, "QOLSR, QoS Routing for Ad Hoc Wireless Networks using OLSR," Wiley European Trans. Telecommunications, vol. 15, no. 4, 2005, pp. 427-42.

[45] C. E. Perkins and P. Bragwat, "Highly Dynamic DestinationSequenced Distance-Vector Routing (DSDV) for Mobile Computers," Proc. ACM SIGCOMM '94, 1994, pp. 234-44.

[46] IEEE Computer Society, Wireless Medium Access Control (MAC) and Physical Layer (PHY) Specifications for High-Rate Wireless Personal Area Networks (WPANs), 2003, IEEE Std. 802.15.3-2003.

[47] D. Johnson, D. Maltz, and J. Broch, DSR: The Dynamic Source Routing Protocol for Multihop Wireless Ad Hoc Networks in Ad Hoc Networking, ch. 5, Addison-Wesley, 2001, pp. 139-72.

[48] F. Kuipers and P. Van Mieghem, "Conditions that Impact the Complexity of QoS Routing," IEEE/ACM Trans. Net., vol. 13, no. 4, pp. 717-30, 2005.

[49] IEEE Computer Society, Wireless LAN Medium Access Control (MAC) and Physical Layer (PHY) Specifications, ANSI/IEEE Std. 802.11, 1999.

[50] C. E. Perkins and E. M. Royer, "Ad hoc On-Demand Distance Vector Routing," Proc. 2nd IEEE Wksp. Mobile Computing Systems and Applications, New Orleans, LA, Feb. 1999, pp. 90-100.

[51] C. Sarr et al., "Improving Accuracy in Available Bandwidth Estimation for IEEE 802.11-Based Ad Hoc Networks," Proc. 3rd IEEE Conf. Mobile Ad Hoc and Sensor Systems, Vancouver, Oct. 2006, pp. 517-20.

[52] L. Hanzo and R. Tafazolli, "Quality of Service Routing and Admission Control for Mobile Ad Hoc Networks with a Con-
tention-Based MAC Layer," Proc. 3rd IEEE Conf. Mobile Ad Hoc and Sensor Systems, Vancouver, Oct. 2006, pp. 501-14.

[53] D. Porcino and W. Hirt, "Ultra-Wideband Radio Technology: Potential and Challenges Ahead," IEEE Commun. Mag., vol. 41, July 2003, pp. 66-74.

[54] S. Roy et al., "Ultra-wideband Radio Design: The Promise of High-Speed, Short-Range Wireless Connectivity," Proc. IEEE, vol. 92, Feb. 2004, pp. 295-311.

[55] P. Jacquet et al., "Optimized Link State Routing Protocol for Ad Hoc Networking," Proc. IEEE Multi Topic Conf., Dec. 2001, pp. 62-68.

[56] G. Aggelou and R. Tafazolli, "RDMAR: A Bandwidth-Efficient Routing Protocol for Mobile Ad Hoc Networks," Proc. 2nd ACM Int. Wksp. Wireless Mobile Multimedia, 1999, pp. 26-33.

[57] H. Schulzrinne et al., "RTP: A Transport Protocol for Real-Time Applications (rfc 3550)," IETF RFC, July 2003.

\section{BIOGRAPHIES}

LAJOS HANZO II [StM'05] (I.hanzo@surrey.ac.uk) graduated with an MEng degree in Computer Engineering from the University of Southampton in 2004. Since October 2004 he has been working towards his PhD in the Centre for Communication Systems Research at the University of Surrey, UK. His research interests include MAC and routing protocols for the provision of QOS in mobile ad hoc networks and wireless sensor networks.

RAHIM TAFAZOLLI [M'89] is a professor of mobile/personal communications and Head of Mobile Communications Research at the Center for Communication Systems Research (CCSR), University of Surrey, UK. He is the editor of Technologies for the Wireless Future, vols. 1 and 2 (2004 and 2006). He is nationally and internationally known in the field of mobile communications and acts as external examiner for the British Telecom M.Sc. course. He has been active in research for more than 20 years and has authored and co-authored more than 300 papers in refereed international journals and conferences. He is a consultant to many mobile companies, has lectured at, chaired and been invited as keynote speaker to a number of IEE and IEEE workshops and conferences. He has been Technical Advisor to many mobile companies and the European Union all in the field of mobile/wireless communications. He is the Founder and past Chair of the IEE International Conference on 3rd Generation Mobile Communications. He is Chair of the EU Expert Group on Mobile Technology Platform, EMobility as well as Chair of the Working Group on Post-IP. 